\title{
State of the Art in BCI Research: BCl Award 2010
}

Christoph Guger ${ }^{1}$, Guangyu Bin², Xiaorong $\mathrm{Gao}^{2}$, Jing Guo ${ }^{2}$, Bo Hong2, Tao Liu², Shangkai Gao², Cuntai Guan³, Kai Keng Ang3, Kok Soon Phua 3 , Chuanchu Wang', Zheng Yang Chin 3 , Haihong Zhang3, Rongsheng Lin 3 , Karen Sui Geok Chua ${ }^{4}$, Christopher Kuah4, Beng Ti Ang5, Harry George ${ }^{6}$, Andrea Kübler 6 , Sebastian Halder ${ }^{7}$, Adi Hösle7, Jana Münßinger 7 , Mark Palatucci ${ }^{8}$, Dean Pomerleau ${ }^{8}$, Geoff Hinton, Tom Mitchell ${ }^{8}$, David B. Ryan ${ }^{9}$, Eric W. Sellers 9 , George Townsend ${ }^{10}$, Steven M. Chase ${ }^{11}$, Andrew S. Whitford ${ }^{12}$, Andrew B. Schwartz ${ }^{11}$, Kimiko Kawashima13, Keiichiro Shindo ${ }^{13}$, Junichi Ushiba13, Meigen Liu'13 and Gerwin Schalk ${ }^{14}$ ${ }^{1}$ g.tec medical engineering GmbH/Guger Technologies OG, Graz ${ }^{2}$ Department of Biomedical Engineering, School of Medicine, Tsinghua University, Beijing ${ }^{3}$ Institute for Infocomm Research, $A^{*}$ STAR ${ }^{4}$ Tan Tock Seng Hospital ${ }^{5}$ National Neuroscience Institute

${ }^{6}$ Department of Psychology I, University of Würzburg IInstitute of Medical Psychology and Behavioural Neurobiology, University of Tübingen ${ }^{8}$ Carnegie Mellon University, Intel Labs, University of Toronto ${ }^{9}$ East Tennessee State University

${ }^{10}$ Algoma University BCI Lab ${ }^{11}$ Dept. of Neurobiology, University of Pittsburgh ${ }^{12}$ Dept. of Bioengineering, University of Pittsburgh

${ }^{13}$ Keio University ${ }^{14}$ Wadsworth Center, Albany

${ }^{1}$ Austria

${ }^{2}$ China 3,4,5Singapore

6,7 Germany $8,9,11,12,14$ USA

${ }^{10}$ Canada ${ }^{13}$ Japan 


\section{Introduction}

The possibility of brain-computer communication based on the electroencephalogram (EEG) has been discussed almost four decades ago (Vidal, 1973). In another pioneering work, Farwell and Donchin described the use of evoked potentials for communication (Farwell, 1988). Up to the early 2000s, no more than 5 groups were active in brain-computer interface (BCI) research. Now, about 200-300 laboratories are focused on this work. This dramatic growth has been driven by high performance and low cost of computing power and related instrumentation, increased understanding on normal and abnormal brain function, and improved methods for decoding brain signals in real time. As a result, the performance and usability of BCI systems have advanced dramatically over the past several years.

BCI systems can be described by the following characteristics: (i) invasive (electrocorticogram (ECoG), spikes) or non-invasive (EEG, NIRS (near infraredspectroscopy), fMRI (functional magnetic resonance imaging), MEG (magnetoencephalogram)) systems (Leuthardt, 2004, Owen, 2008, Velliste, 2008, Wolpaw 2003, Pfurtscheller 2010a, 2010b), (ii) portable (EEG) or stationary (fMRI, ECoG, spikes), (iii) according to application area (spelling, wheelchair control, brain painting, research,...) (Sellers, 2010, Galán, 2008, Kübler, 2008), (iv) type of BCI principle used (P300, SSVEP (steady-state visual evoked potential)), steady state evoked potential (steady-state somatosensory evoked potential)), motor imagery, slow cortical potentials (Bin, 2009, Birbaumer, 2000, Pfurtscheller, 2010, Krusienski, 2006) (v) speed and accuracy, (vi) training time and reliability, (vii) synchronous and asynchronous, (viii) low cost (EEG, NIRS) and high costs (MEG, fMRI, spikes), (ix) degrees of freedom. A detailed review can be found in Allison (Allison, 2007). Over the last years the importance of specific properties changed, new technologies were developed that enabled new applications or made BCI systems affordable. For example, in the late 90 s there were just a few real-time systems worldwide. At present, almost every lab is equipped with real-time BCI systems.

To highlight these trends and developments of BCI technology, g.tec began to sponsor an annual BCI Award in 2010. The prize, endowed with 3,000 USD, is an accolade to recognize outstanding and innovative research in the field of brain-computer interface research and application. Each year, a renowned research laboratory is asked to judge the submitted projects and to award the prize. The jury consists of world-leading BCI experts recruited by the awarding laboratory. g.tec is a leading provider of BCI research equipment and has a strong interest in promoting excellence in the field of BCI to make BCIs more powerful, more intelligent and more applicable. The competition is open to any BCI group worldwide. There is no limitation or special consideration for the type of hardware or software used in the submission. This year, the jury was recruited by its chair Dr. Gerwin Schalk of the Wadsworth Center in Albany, New York. It consisted of world-leading experts in the BCI community: Theresa Vaughan, Eric Sellers, Dean Krusienski, Klaus-Robert Mueller, Benjamin Blankertz, and Bo Hong.

The jury scored the submitted projects on the basis of the following criteria:

- does the project include a novel application of the BCI?

- is there any new methodological approach used compared to earlier projects?

- $\quad$ is there any new benefit for potential users of a BCI?

- $\quad$ is there any improvement in terms of speed of the system (e.g., bits/min)?

- $\quad$ is there any improvement in system accuracy?

- does the project include any results obtained from real patients or other potential users? 
- $\quad$ is the used approach working online/in real-time?

- $\quad$ is there any improvement in terms of usability?

- does the project include any novel hardware or software developments?

We received a total of 57 high quality submissions. Out of these submissions, the jury nominated the 10 top-ranked candidates for the BCI Research Award in April 2010 (see Table 1). The following sections describe each project in more detail.

\begin{tabular}{|c|c|}
\hline Name and institution & Title of BCI project \\
\hline $\begin{array}{l}\text { Guangyu Bin, Xiaorong Gao, Shangkai } \\
\text { Gao }\end{array}$ & $\begin{array}{l}\text { A high-speed word spelling BCI system } \\
\text { based on code modulated visual evoked } \\
\text { potentials }\end{array}$ \\
\hline $\begin{array}{l}\text { Cuntai Guan, Kai Keng Ang, Kok Soon } \\
\text { Phua, Chuanchu Wang, Zheng Yang } \\
\text { Chin, Haihong Zhang, Rongsheng Lin, } \\
\text { Karen Sui Geok Chua, Christopher } \\
\text { Kuah, Beng Ti Ang }\end{array}$ & $\begin{array}{l}\text { Motor imagery-based brain-computer } \\
\text { interface robotic rehabilitation for stroke }\end{array}$ \\
\hline Jing Guo, Shangkai Gao, Bo Hong & $\begin{array}{l}\text { An active auditory } \mathrm{BCI} \text { for intention } \\
\text { expression in locked-in }\end{array}$ \\
\hline Tao Liu, Shangkai Gao, Bo Hong & $\begin{array}{l}\text { Brain-actuated Google search by using } \\
\text { motion onset VEP }\end{array}$ \\
\hline $\begin{array}{l}\text { Harry George, Sebastian Halder, Adi } \\
\text { Hösle, Jana Münßinger, Andrea Kübler }\end{array}$ & Brain Painting - "Paint your way out" \\
\hline $\begin{array}{l}\text { Mark Palatucci, Dean Pomerleau, Geoff } \\
\text { Hinton, Tom Mitchell }\end{array}$ & $\begin{array}{l}\text { Thought recognition with semantic output } \\
\text { codes }\end{array}$ \\
\hline David B. Ryan and Eric W. Sellers & $\begin{array}{l}\text { Predictive spelling with a P300-base BCI: } \\
\text { increasing communication rate }\end{array}$ \\
\hline George Townsend & $\begin{array}{l}\text { Innovations in P300-based BCI stimulus } \\
\text { presentation methods }\end{array}$ \\
\hline $\begin{array}{l}\text { Steven M. Chase, Andrew S. Whitford, } \\
\text { Andrew B. Schwartz }\end{array}$ & $\begin{array}{l}\text { Operant conditioning to identify } \\
\text { independent, volitionally-controllable } \\
\text { patterns of neural activity }\end{array}$ \\
\hline $\begin{array}{l}\text { Kimiko Kawashima, Keiichiro Shindo, } \\
\text { Junichi Ushiba, Meigen Liu }\end{array}$ & $\begin{array}{l}\text { Neurorehabilitation for chronic-phase stroke } \\
\text { using a brain-machine interface }\end{array}$ \\
\hline
\end{tabular}

Table 1. Nominees of the BCI Award 2010.

\section{Nominated projects}

\section{Project 1: A high-speed word spelling BCI system based on code modulated visual} evoked potentials

Guangyu Bin, Xiaorong Gao, Shangkai Gao

A high-speed word spelling brain-computer interface (BCI) based on code modulated visual evoked potentials (c-VEPs) was developed. The c-VEP BCI uses a binary pseudorandom sequence for stimulus modulation (Sutter, 1992, Bin, 2009). In the system, the stimuli were set to two states: "light" and "dark", and so a binary sequence can be used as modulation 
sequence. "Light" and "dark" represented " 1 " and " 0 " in the modulation sequence. For instance, the stimulus modulated by a sequence "100010001000..." represented $15 \mathrm{~Hz}$ flickering, when the refresh rate of the monitor was $60 \mathrm{~Hz}$.

The targets of the stimuli are distributed as an array and the number of targets can be selected to be either $16(4 \times 4), 32(4 \times 8)$ or $64(8 \times 8)$. Figure 1 A shows a c-VEP based BCI system with 16 targets. Each target was periodically modulated by a binary m-sequence. Except for a fixed time lag between two consecutive targets, the used modulation sequences in one period were the same. As an example, Figure 1B presented the modulation sequences of a c-VEP system with sixteen targets. In the system, a binary m-sequence with 63 elements and its time shift sequences are used as the modulation signals, and there is a four-frame lag between two consecutive targets.

A template matching method is used for target identification. In the training stage, the user is instructed to fixate on one of the targets (such as target " 10 ", the training target), and the template of the training target is obtained. According to the time lag, templates of all targets are generated. In the online application, the correlation coefficient between EEG data and every template is calculated. If the coefficient remains above a certain threshold and is larger than all the others for a certain amount of time, then the corresponding target is considered to be the selected one.

The stimulus was presented on a CRT monitor with $60 \mathrm{~Hz}$ refresh rate and EEG data were recorded with a g.USBamp amplifier (g.tec medical engineering GmbH, Austria). The parallel port is used to synchronize EEG data acquisition with the stimulus. The system is implemented with EEGOnline, which is a general-purpose system for real-time EEG signal processing developed by Tsinghua University. EEGOnline provides a comprehensive framework of functionalities that allows the user to focus on the implementation of his application specific module. It can be used for EEG data acquisition, brain-computer interface research and brain monitoring applications.

A

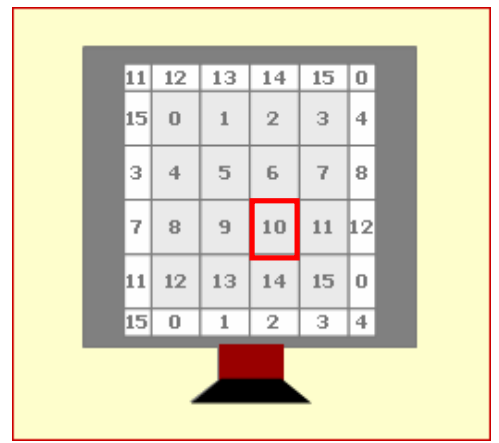

B

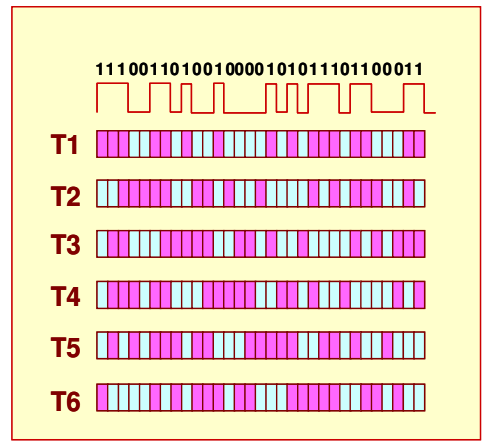

Fig. 1. (A) The target arrangement of the c-VEP based BCI. The sixteen targets are distributed in a $4 \times 4$ array surrounded by a border to eliminate the effect of the array boundary. When the border fields are stimulated according to the wrap-around principle, all targets have equivalent neighbours. Thus, the responses obtained when the subject fixated on different targets were practically identical. (B) The modulation sequences of the targets in one stimulation cycle. Each sequence is from a binary $\mathrm{m}$-sequence. There is a fourframe lag between two consecutive sequences. All targets were activated simultaneously, and the stimulation cycle was repeated constantly. 
For the 16 targets system, twelve healthy right-handed adults with normal or corrected-tonormal vision served as volunteers after giving informed consent. The information transfer rate (ITR) averaged more than $92.8 \mathrm{bits} / \mathrm{min}$. Moreover, a system with 32 possible selections was used for spelling. These thirty-two possible selections corresponded to the 26 characters of the alphabet and another six keys (SPACE, DELETE, ENTER, three punctuators) are presented on the screen. This system was tested with many users and the achieved spelling speed was approximately 15-20 characters per min.

The main advantages of the c-VEP based BCI system include high-speed communication, more targets, and lower user variation.

\section{Project 2: Motor imagery-based brain-computer interface robotic rehabilitation for stroke}

\section{Cuntai Guan, Kai Keng Ang, Karen Sui Geok Chua, Beng Ti Ang, Kok Soon Phua,}

Christopher Kuah, Chuanchu Wang, Zheng Yang Chin, Haihong Zhang, Rongsheng Lin

Stroke is the leading cause of severe disabilities in the developed world (Beers, 2000). Each year, there are around 15 million new stroke cases worldwide. About $30 \%$ of stroke survivors need various forms of rehabilitation. Among these, upper limb weakness and loss of hand function are among the most devastating types of disabilities. Despite optimal acute medical treatment and modern rehabilitation, $45 \%$ of the patients do not achieve complete recovery of their bodily functions. In addition, $85 \%$ to $90 \%$ of stroke survivors with upper limb impairment do not regain full functional use of their upper extremities. Limitations in current physiotherapy and occupational therapy techniques include: (i) difficulties in rehabilitation for the severely paralyzed arm and hand which are often treated with passive modalities, (ii) difficulties in achieving intensive rehabilitation and high repetitions in those with moderate to severe upper extremity paralysis, (iii) problems in motivating and sustaining patient interest in repetitive exercises, (iv) therapy is often perceived to be boring due to lack of immediate biofeedback.

Recently, robot-aided rehabilitation has been clinically investigated worldwide to try to address these issues. Despite continuous improvements and progress in the field, there is a strong request from rehabilitation clinicians to call for more efficacious and more targetspecific approaches to address the aforementioned issues.

Given recent progress in BCI technologies, there is an increased interest in applying BCI to stroke rehabilitation (Daly, 2008, Wolpaw, 2002, Ang, 2009, Ang, 2010), as BCIs provide a direct and real-time link between the human brain (in particular, cortical area) to external devices (Birbaumer, 2006, Ang, 2008). With this motivation, we embarked on this project from April 2007 to Oct 2009. Our hypotheses for this project were as follows:

1. A BCI provides an effective guide and visual feedback to motivate patients

2. BCI guides patients to improve the excitation of the motor cortex

3. Mechanical stimulation provides movement training, as well as motor/sensory feedback to the patient

4. Combination of BCI and mechanical stimulations could provide an effective guided training system

The system was developed and evaluated with patients as depicted in Figure 2. It consisted of a BCI and a robotic arm (MANUS from InMotion). The patient was asked to perform motor imagery (instead of movement, in order to prevent possible use of compensated movements due to mal-adaptation after stroke insult). Once the $\mathrm{BCI}$ detected motor imagery (with a technique developed in our group, which is the winning algorithm in BCI Competition IV 2008, dataset II (Calautti, 2003)), it triggered the robotic arm to move the 
patient's arm to a designated direction. The direction was randomly selected by the training protocol.

A clinical trial (Registration number NCT00955838 in ClinicalTrials.gov) was then performed to assess the effects. As a reference, we also recruited patients to use the MANUS alone for rehabilitation. 26 patients were recruited, and randomized into two groups (15 in MANUS group, 11 in BCI group).

Patients performed rehabilitation training for 4 weeks, 3 sessions per week, and each session lasted around 1 hour. The clinical evaluation was done at the beginning of the training (week 0), mid of the training (week 2), and at the end of the training (week 4). A follow-up assessment was performed at week 12 . However, due to the nature of the training process, patients in the MANUS group performed 960 repetitions, while the BCI group only performed 160 repetitions, i.e., a 1:6 difference in intensity.

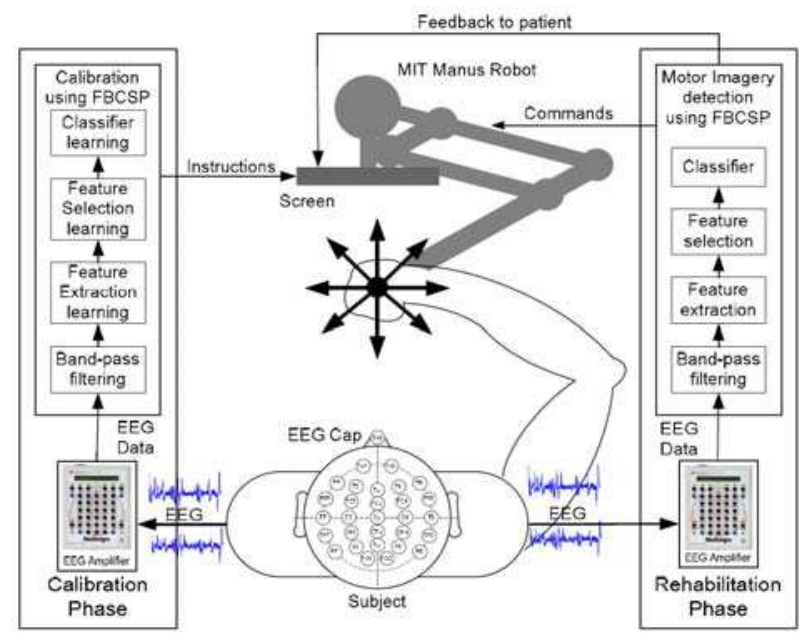

A

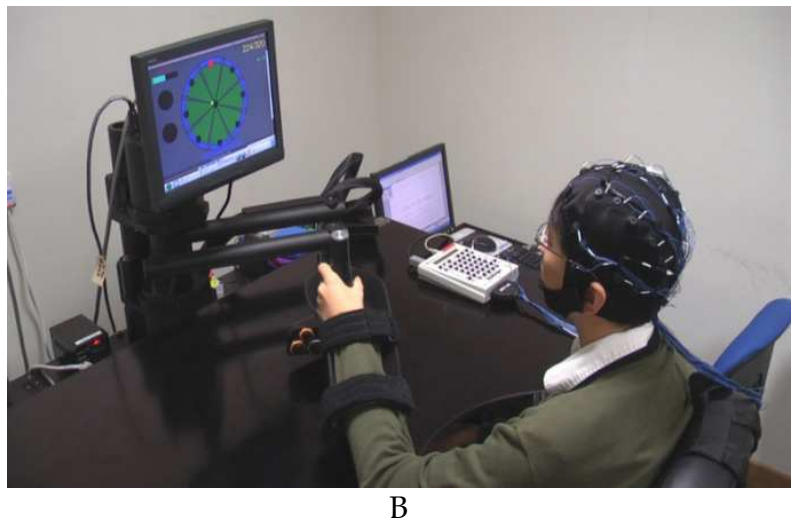

Fig. 2. (A) Block diagram of the motor imagery BCI with robotic rehabilitation. (B) Actual system tested in hospital by patients. 
Table 1 summarizes the outcome of our study and reports the Fugl-Meyer Assessment (FMA) score. The FMA score demonstrates an overall recovery of motor impairment. The results show that, at week $4 \& 12$, the improvements for the BCI and the MANUS groups are statistically significant. Encouragingly, for both groups, it seems that the improvement is maintained after 12 weeks - a suggestion for sustainability of the rehabilitation training. There is no significant difference in improvement between the two groups.

\begin{tabular}{|l|l|l|l|l|l|l|l|l|}
\hline & \multicolumn{9}{|l|}{ BCI group } & \multicolumn{2}{l|}{ MANUS group } \\
\hline & Week 0 & Week 2 & Week 4 & Week12 & Week 0 & Week 2 & Week 4 & Week12 \\
\hline $\begin{array}{l}\text { Mean FMA } \\
\text { score } \pm \text { STD }\end{array}$ & $26.3 \pm 10.3$ & $27.4 \pm 12.0$ & $30.8 \pm 13.8$ & $31.5 \pm 13.5$ & $26.6 \pm 18.9$ & $29.9 \pm 20.6$ & $32.9 \pm 21.4$ & $33.9 \pm 20.2$ \\
\hline $\begin{array}{l}\text { Improvement } \\
\pm \text { STD }\end{array}$ & - & $1.1 \pm 4.1$ & $4.5 \pm 6.1$ & $5.3 \pm 6.3$ & - & $3.2 \pm 4.5$ & $6.2 \pm 6.3$ & $7.3 \pm 9.4$ \\
\hline t-test p value & - & 0.402 & 0.032 & 0.020 & & 0.020 & 0.003 & 0.013 \\
\hline
\end{tabular}

Table 1. Mean FMA score, improvement related to week 0 and paired t-test for BCI and MANUS groups.

In this project, we evaluated the feasibility of using a BCI for stroke rehabilitation. Some of the results are summarized as follows:

- There is evidence from this study to suggest that motor imagery rehabilitation for stroke using the $\mathrm{BCI}$ is as effective and comparable to robotic rehabilitation, while the $\mathrm{BCI}$ group needs much less intensity compared to robotic training (a factor of 6 ).

- Stroke patients are able to use BCI to perform rehabilitation (we did a pre-screening with 54 patients; around $89 \%$ of the patients, who were all naïve users, can operate a BCI with an accuracy better than chance level).

- $\quad$ BCI based on automatic feature selection and band-pass filtering was proven to be reliable across various patients.

- The combination of the BCI and the robotic arm seemed a feasible setup.

Invaluable experience was gained throughout the project and it must be noted that not much literature exists about BCI and stroke. The following important issues will be considered in our follow-up study:

- $\quad \mathrm{BCI}$ is considered as a guide for patients. However, what is the best way to detect motor activities?

- Should the detection make use of the whole brain EEG or just from the lesion hemisphere?

- Should the detection strategy change over time when patients get more recovery?

- How to balance the detection accuracy and patients' motivation, especially at the early stage of the training, when the patient is not good at exercising motor imagery?

- Which types of patients are most suitable to use the BCI (versus robotic, etc.)?

\section{Project 3: An active auditory BCI for intension expression in locked-in}

Jing Guo, Shangkai Gao, Bo Hong

An intention expression system using event-related potentials (ERP) elicited by sound stimulus is presented. It allows subjects to express their intentions by mentally selecting a target among a random sequence of sound options. BCIs based on visual modalities have proved to be highly effective, but too limited for those locked-in patients who have compromised vision. Since hearing is usually preserved in severely paralyzed patients, a 
novel auditory BCI paradigm using subjects' active mental response is implemented in this study.

An auditory stimulus was given in form of a sequence of 5 spoken digits in Chinese, i.e., $1,2,3,4,5$, presented randomly and repeatedly. Each digit can be used to represent one possible option of intentions. The gender of the voices was random. A segment of the voice sequence is shown in Figure 3. The subject operated this system by focusing on the target digit voices and silently telling whether the target digit was a male or female voice. The subject' voluntary mental response to target digit voices elicited a distinct ERP over the centro-parietal cortex, which was quite different from that of the non-targets.

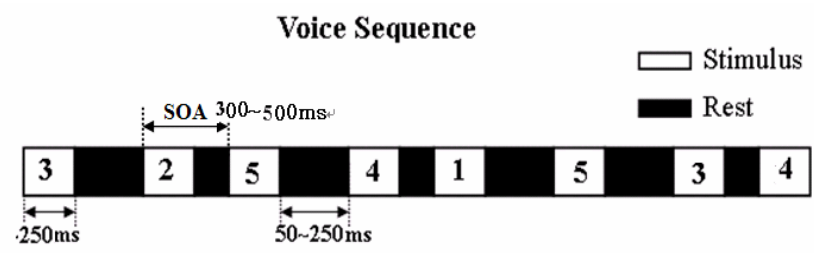

Fig. 3. Auditory BCI scheme with voice sequence design

Figure 4 shows twelve subjects' grand averaged temporal waveform and amplitude topographic maps. It revealed a negative deflection (N2) with latency of $100-300 \mathrm{~ms}$ and peak at $120 \mathrm{~ms}$, which displays more negativity in the target item. A broad late positive component (LPC) between $400-700 \mathrm{~ms}$ was elicited by the target with a parietal topography maximum around $\mathrm{Pz}$, and was absent in response to non-target stimuli.
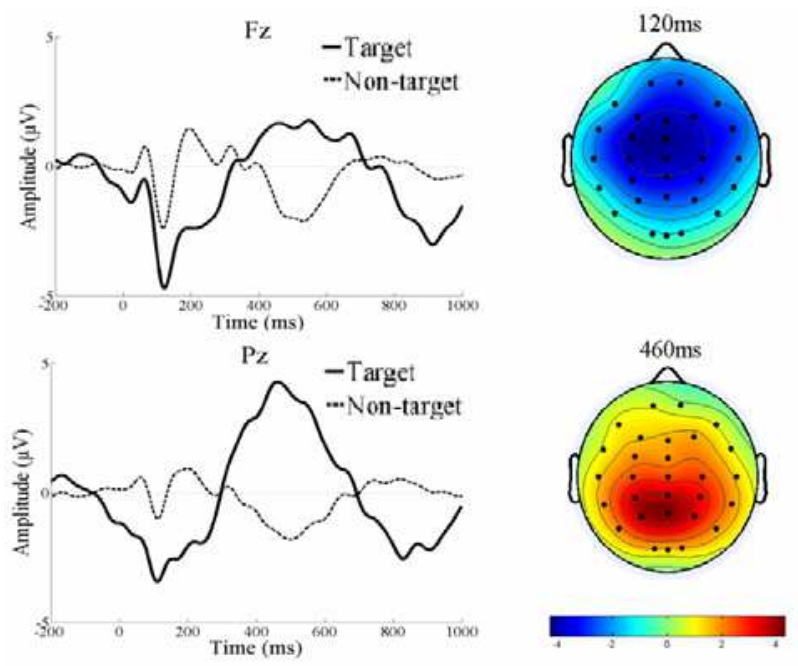

Fig. 4. Averaged temporo-spatial pattern of the ERP.

The proposed paradigm shares some of its features with P300-based BCIs, e.g., the 'oddball' design of the stimulus sequence. However, in the current paradigm, voluntary mental tasks were employed to enhance the LPC response, which may involve more 'active' mental processes than the $\mathrm{P} 300$ paradigm. 


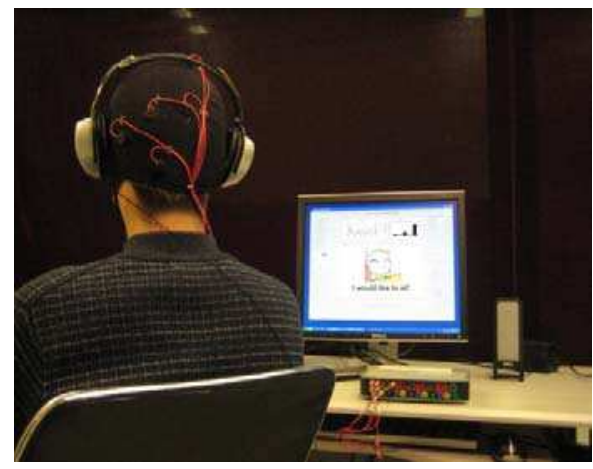

Fig. 5. BCI system configuration

The auditory stimulus in the online system is still a spoken Chinese digits of 1-5, which is used to represent five possible options of intentions, e.g., "cold", "hot", "eat", "drink" and "sit". After signal processing and target detection of the ERP, the system returns the subject's choice result visually and verbally, to help the subject express his current intentions. The illustration of online application system is shown in Figure 6. The voice sequence is presented to the subject by headphones. EEG is recorded from less than 3 electrodes (optimally selected for individuals) using the g.USBamp amplifier (g.tec medical engineering $\mathrm{GmbH}$, Austria). The whole system is running under MATLAB.

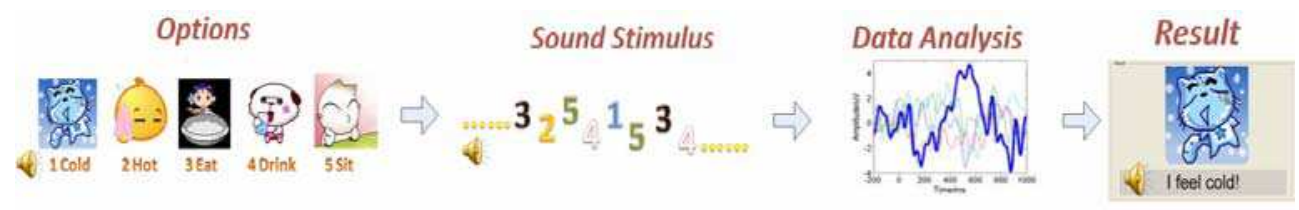

Fig. 6. Principle of the BCI system
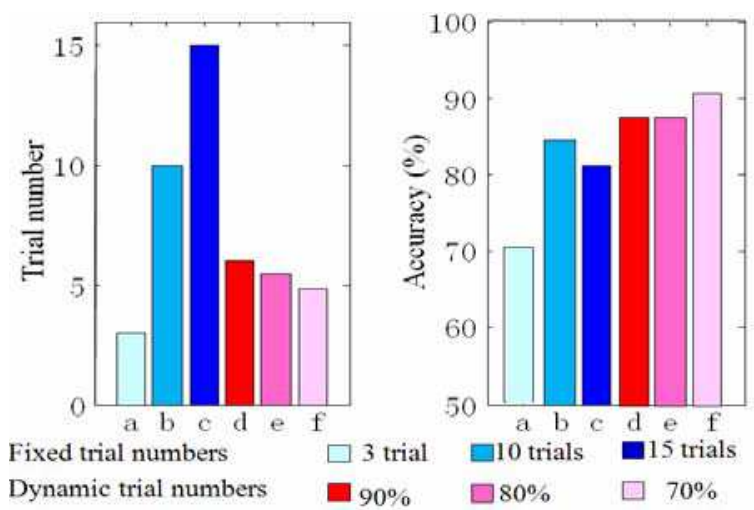

Fig. 7. Accuracy of BCI systems

In our system, a statistical approach is proposed to adaptively adjust the number of trials to be averaged for a decision. The discriminant function is computed with a support vector 
machine algorithm at each sample; its result was converted into probabilities of each BCI choice being the target. If the highest probability among all $\mathrm{BCI}$ choices reached a predefined threshold that was estimated from training accuracy, the adaptive algorithm terminated and selected the target with the highest probability.

Figure 7 depicts the averaged online results of 8 subjects, using fixed or dynamic trial numbers. The trial numbers of the fixed method were set to 3, 10 and 15 . The thresholds of the dynamic method were set as $90 \%, 80 \%$ and $70 \%$ of training accuracy. There were about 4-6 trials to reach the equivalent accuracy of 15-trial averaging, demonstrating the advantage of this adaptive approach.

This adaptive active auditory BCI system allows the subjects to express their intentions, which is potentially helpful for the locked-in patients with compromised vision or the ALS patients.

\section{Project 4: Brain-actuated Google search by using motion onset VEP}

Tao Liu, Shangkai Gao, Bo Hong

The motion-onset VEP (mVEP), a scalp potential from the higher visual system for visual motion processing, is promising for BCI applications due to its large amplitude, stable latency, and immunity to low contrast and illumination. In this study, mVEP was used to implement a single-channel brain-computer interface system for brain-actuated Google search. With a flexible and non-flashing interface, mVEP-based BCI system was embedded into screen elements, such as menus and buttons, to achieve a better human computer interaction.

First a vertical bar appears in sequence and moved leftwards in each of the virtual buttons (Figure 8). Users focus on the vertical bar in the desired button to operate the system. The motion of the vertical bar elicits the mVEP over the temporo-occipital and parietal cortex, areas responsible for visual motion processing. Figure 8A shows a virtual keypad interface, composed of 6 virtual buttons, to type in the search terms. With the dynamic menu in the virtual keypad, 26 letters together with another 10 symbols were divided into 6 groups, thus making character selection a two-step process. Furthermore, the web browser was modified with an embedded virtual command toolbar (Figure 8B) to enable the user to directly

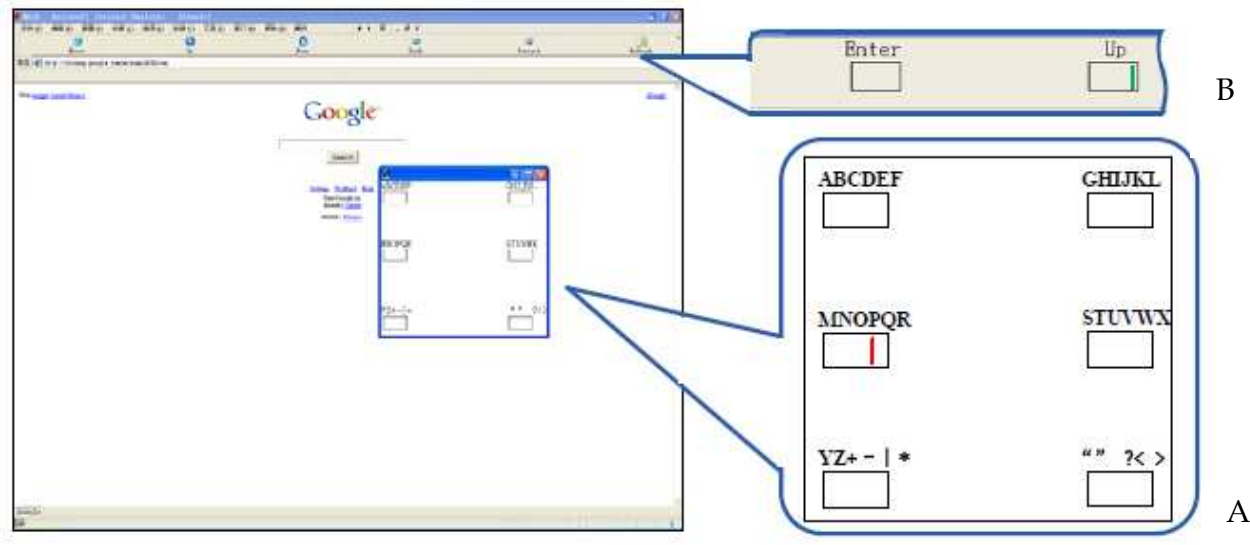

Fig. 8. Screen shot from the BCI system to operate Google. 
interact with the web contents and accomplish web-browsing tasks, such as "Back", "Forward", and "Enter".

The grand average temporal waveform of the mVEP and its amplitude topographic maps are shown in Figure 9. Similar to previous findings, it is characterized by a negative N2 component with asymmetrical occipito-temporal topography around $200 \mathrm{~ms}$ and a positive complex of P2 and P3 component, which has a similar spatial distribution with N2 component, but with an additional broader parietal distribution at $325 \mathrm{~ms}$.

Using motion-onset VEP, the proposed BCI system requires no flashing or sudden change of visual objects, which poses no discomfort and less visual fatigue on BCI users. Additionally, because of the tolerance to a large contrast range, the $\mathrm{mVEP}$ is a steady and robust signal for a highly adaptable BCI system that could work in various applications. Furthermore, the localized spatial distribution of the mVEP (Figure 9) allows to perform the target selection with fewer channels. By contrast, in the previous P300-based BCI systems, the involvement of more channels is needed to improve the classification accuracy due to the relatively broad distribution of P300 component. In a practical online BCI system, fewer channel means less preparation time and lower system cost.

To minimize the number of required EEG channels, the squared Pearson product-moment coefficient $\left(\mathrm{r}^{2}\right)$ between the EEG channel and the task was calculated. Then we sorted the channels by their $\mathrm{r}^{2}$ value. Since the EEG features relevant to the motion stimuli are localized, we selected only the channel with highest $r^{2}$ value for each subject (typically at P3, P7 or O1 electrode).

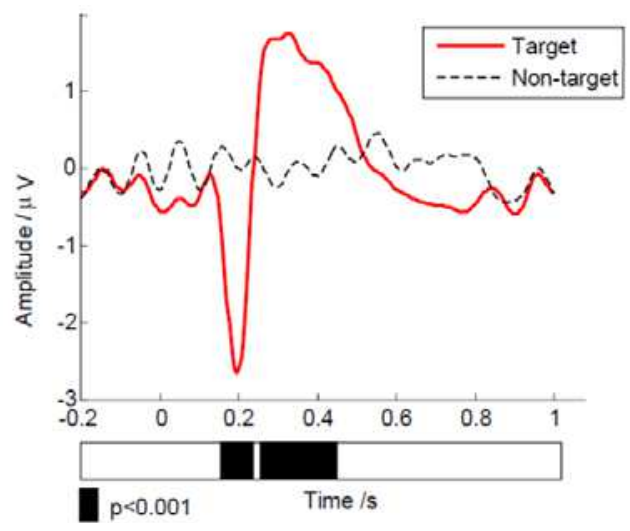

A
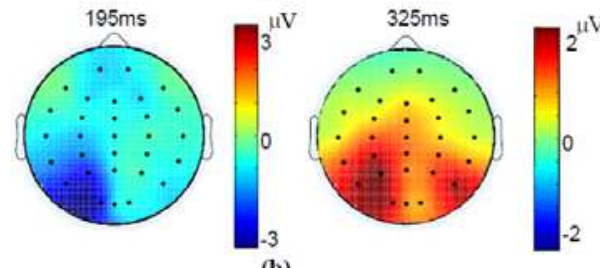

(b)

B

Fig. 9. Temporal (A)-spatial (B) patterns of mVEP

12 subjects were instructed to input the search the term "BCI", then search and select the desired link. The EEG epochs from 'best' electrodes were windowed from $100 \mathrm{~ms}$ to $500 \mathrm{~ms}$ following the motion-onset and down-sampled to $20 \mathrm{~Hz}$ to form a 9-point feature vector. The support vector machine was used for the target detection in the online application. The system has been tested with both the g.tec and Neuroscan amplifiers. As shown in Table 2, all the subjects could successfully operate this system, and completed the task in a reasonable time ranging from $50.9 \mathrm{~s}$ to $234.2 \mathrm{~s}$. A mean ITR of $42.1 \mathrm{bits} / \mathrm{min}$ was achieved by 12 subjects, with an average accuracy of $91 \%$. 


\begin{tabular}{lllll}
\hline Subject & Operations & Trials/ Operation & Accuracy $\mathbf{( \% )}$ & Time(sec) \\
\hline S1 & 14 & 2.4 & 92 & 79.4 \\
S2 & 10 & 4.3 & 100 & 77.3 \\
S3 & 18 & 5.6 & 89 & 164 \\
S4 & 26 & 5.5 & 77 & 234.2 \\
S5 & 22 & 6.0 & 86 & 210.1 \\
S6 & 18 & 5.3 & 89 & 159.6 \\
S7 & 11 & 2.4 & 91 & 61.6 \\
S8 & 14 & 4.7 & 93 & 114.6 \\
S9 & 11 & 2.9 & 91 & 68.2 \\
S10 & 14 & 3.2 & 93 & 91.5 \\
S11 & 10 & 1.9 & 100 & 50.9 \\
S12 & 14 & 4.9 & 93 & 117.9 \\
Average & $\mathbf{1 5 . 1}$ & $\mathbf{4 . 1}$ & $\mathbf{9 1}$ & $\mathbf{1 1 9 . 1}$ \\
\hline
\end{tabular}

Table 2. BCI Accuracy

The first online BCI system using non-flashing VEP is presented here. The Google search application was successfully implemented and tested on 12 subjects with only 1 EEG channel.

\section{Project 5: Brain painting - BCI meets patients and artists in the field}

\section{Harry George, Sebastian Halder, Adi Hösle, Jana Münßinger, Andrea Kübler}

Current BCI systems have primarily been developed to replace the lost abilities of patients diagnosed with motor-neuron diseases such as amyotrophic lateral sclerosis (ALS). Of these lost abilities, the most important seems to be that of communication, represented by the increasing volume worldwide of research and development into such applications. Another valuable element of human life, however, is that of creative expression. Through modification of the P300-BCI communication system it was possible to create an application that provides the ability for such expression. We call this Brain Painting.

The P300-Brain Painting application is a new online BCI-application created from the collaboration between artist Adi Hösle and the Universities of Würzburg and Tübingen. Based on the P300 elicited by a rare event in an oddball paradigm (Farwell, 1988), it enables users to express themselves, not only verbally, but also creatively through picture painting. Replacement of the matrix of the P300-Spelling application with a new painting matrix containing functions such as cursor control, shape, object size, grid size and color selections guarantees individual selection of objects and placement on the canvas. While the rows and columns of the matrix start flashing in random order, the user has to concentrate on the symbol of the desired function, which elicits a P300 that is detected and translated by the device. Different objects can be "drawn" on a virtual canvas to produce images of an abstract nature (see Figure 10).

A first evaluation of the P300-Brain Painting application (Muenssinger, 2010) demonstrated a high accuracy in ALS-patients (above $89 \%$ in two of the three patients) when reproducing existing paintings. This accuracy was even higher than in healthy controls. This is outstanding because other research found paralyzed patients to show lower performance in P300-BCI use than healthy controls (Piccione, 2006). Moreover, we further increased the accuracy by implementing a black and white matrix for painting that turned out to be less distracting than the colored matrix. Accuracy of the P300 black and white Brain Painting 
matrix equalled that achieved using the P300-Spelling application (spelling: $93.20 \%$ (SD \pm 7.50); painting: $92.60 \%$ (SD \pm 5.70 ); (Muenssinger, 2010) in healthy subjects.

Qualitative responses from patients using the system were very positive and enthusiastic and they confirmed that they experience satisfaction and are entertained while using the application. They display a strong repeated desire to re-use the system; one patient reported that she was so excited about painting that she spent the whole night planning her next picture. To date, patients using the system have produced numerous images from independent sittings lasting upwards of 1.5 - 3.5 hours. Patients have demonstrated a high motivation, exceeding 8.5 on a visual analogue scale (VAS) ranging from 1 to 10 before and after painting sessions. This serves as an indication that participants like the application, find it intuitive, highly user-friendly, and enjoy working with it.

Applied as leisure time activity, the P300-Brain Painting application provides patients with the ability to be productive again and to participate in prolific society through art exhibitions of their paintings such as that taken place in the Künstlerbund in Tübingen (a German artist association) in November 2007 (Kübler, 2008). Recently Brain Painting has been showcased to several healthy prominent German artists as an assessment by healthy subjects. The application was received enthusiastically, demonstrated and evaluated for further improvements.

The Brain Painting application serves to satisfy some basic human needs, providing a positive, useful difference and great enthusiasm to ALS-patients but also as an advanced tool and research platform whereby new technological prototypes and developments in stimulus presentation, online data processing and prototype classifying techniques may be effectively trialled using motivated ALS-patients as subjects. Moreover, pictures produced by ALS-patients and healthy subjects alike have clearly demonstrated that Brain Painting is a new dimension of art that represents a real chance to minimise the gap between healthy subjects and patients through collaborative work in the field of art.
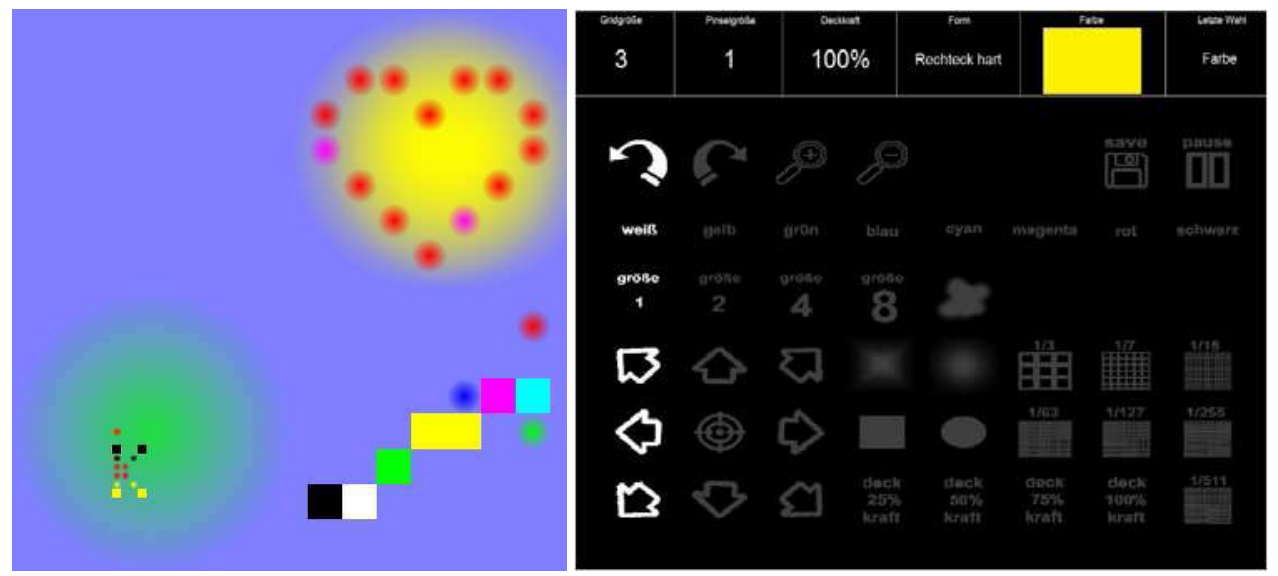

Fig. 10. Image produced by an ALS patient using the P300-Brain Painting application. Left: The painter dedicated the heart in the upper right corner to his wife. Right: Spelling matrix.

\section{Project 6: Thought recognition with semantic output codes}

Mark Palatucci, Dean Pomerleau, Geoff Hinton, Tom Mitchell

Our research focuses on thought recognition, where the objective is to determine the word that a person is thinking about from a recorded image of that person's neural activity. While 
most BCI work to date has focused on control problems using EEG or implanted electrodes, our work focuses on identifying specific words a person is thinking about using higherresolution brain scanners like fMRI and MEG. Our goal is to develop a vocal prosthesis that would allow a person to speak without any movement of his/her body. This could have a major impact, not only on the way people interact with computers, but also on the quality of life of disabled persons.

While machine learning and pattern recognition methods have already made a large impact on this field, most prior work has focused on word category studies with small numbers of categories and moderate amounts of training data. In our research, however, we focus on thought recognition in a limited data setting, where there may not be training examples for every possible word we might want to classify, and the number of possible words can be in the thousands.

Our most recent work was recently published at the Neural Information Processing Systems (NIPS) conference in Vancouver (Palatucci, 2009). In this work, we've made two significant advances to the field of thought recognition and brain-computer-interfaces:

1. Our work has shown that it is possible to predict specific words a person is thinking about with accuracy far above the chance level, even when the classifier is forced to choose from a very large set (e.g. 1,000) of possible words. Thus, we have shown that it is possible to predict a person's mental state at a granularity much higher than previously thought.

2. We have shown that it is not necessary to have training examples for every word we wish to classify. We achieved this by developing a technique known as zero-shot learning with semantic output codes which we expect will have a major impact on the broader fields of pattern recognition and brain-computer-interfaces.

\begin{tabular}{llllllll}
\hline Bear & Foot & Screwdriver & Train & Truck & Celery & House & Pants \\
$(1)$ & $(1)$ & $(1)$ & $(1)$ & $(2)$ & $(5)$ & $(6)$ & $(21)$ \\
bear & foot & screwdriver & train & jeep & beet & supermarket & clothing \\
fox & feet & pin & jet & truck & artichoke & hotel & vest \\
wolf & ankle & nail & jail & minivan & grape & theater & t-shirt \\
yak & knee & wrench & factory & bus & cabbage & school & clothes \\
gorilla & face & dagger & bus & sedan & celery & factory & panties \\
\hline
\end{tabular}

Table 3. The top five most likely words predicted for a held-out fMRI image collected for the word in bold. The number in the parentheses contains the rank of the correct word selected from 941 concrete nouns in English. Note that no training images for the held-out word appeared in the training set.

The problem of thought recognition sits at the intersection of brain-computer interfaces and computational neuroscience. These fields are deeply interrelated, and we believe our research to date has already made contributions to each of these areas.

Regarding brain-computer-interfaces, we have taken steps towards a non-invasive, highbandwidth, brain-computer-interface (BCI). Our results in Table (3) show that we can often predict a specific word that a person is thinking about from a large set of 941 words. This is a much higher granularity of word classification than previously thought possible. Another major feature of this result is that we are able to predict words even when we never saw examples of those words during classifier training. 
The key insight that made these results possible was the notion of a semantic output code. The idea is that the brain encodes meaning of words and objects according to semantic properties such as: is it big? can it be held? does it provide shelter? is it edible? Rather than trying to predict words directly, we try to predict the semantic properties of a word given a fMRI image of neural activity recorded while the person is thinking about the word (see Figure 11). Given a prediction of semantic properties, we can look up in a knowledge base which words have the closet semantic properties to the prediction. A key benefit of this approach is that we no longer need to have training examples for every word we wish to classify. We only need enough training examples in order to classify the individual semantic properties.

We have used computational methods to evaluate different sets of semantic properties for neural activity and believe our findings have made important contributions to the field of computational neuroscience as well.
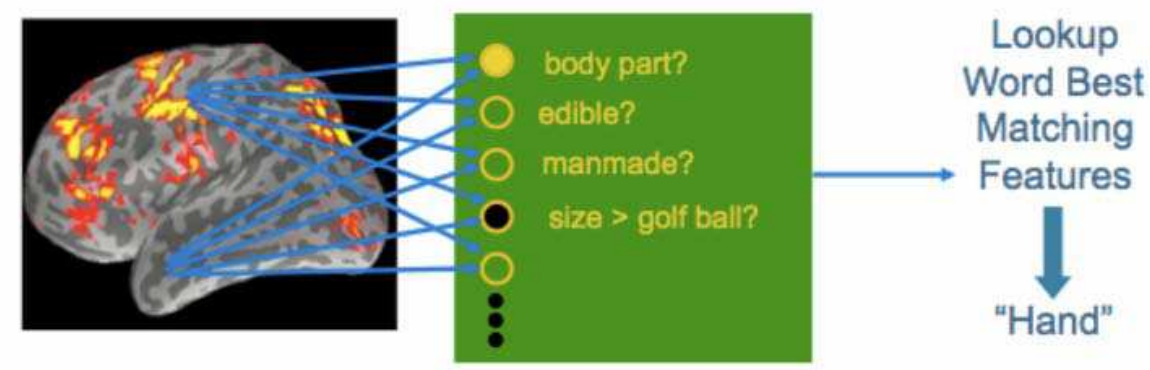

Fig. 11. Example of a Semantic Output Code for the word hand. Rather than predicting words directly, we try to predict semantic properties of the word a person is thinking about given a fMRI image of neural activity. We can then compare the prediction of semantic properties to known words in a semantic knowledge base for many words.

\section{Project 7: Predictive spelling with a P300-based BCI: Increasing communication rate}

David B. Ryan, Eric W. Sellers

Brain-computer interface $(\mathrm{BCI})$ technology can be valuable for people with severe neuromuscular disabilities. The P300-BCI uses the electroencephalogram (EEG), and can return communication to people locked-in by ALS (Townsend, 2010, Sellers 2007, 2010); it requires little training (Guger, 2009) and speed/accuracy is relatively high compared to other BCI systems. However, current communication rate is still a major factor that is limiting widespread BCI use. The current study examines the affect of predictive spelling on P300-BCI performance in terms of output speed/accuracy, and waveform morphology.

Twenty-four subjects participated in the study. None had prior predictive spelling experience. All subjects performed a session with a predictive spelling program and a session without predictive spelling, counter-balanced. Using an 8x9 matrix of alphanumeric characters and commands, the subjects' task was to accurately (i.e., correcting errors) copy a sentence that consisted of 58 items. Each session began with a no-feedback calibration phase of 36 item selections to serve as training data for a step-wise linear discriminant analysis (SWLDA) (Krusienski, 2008) that was then applied online for character classification during the copy task. Items were flashed in quasi-random groups (Townsend, 2010) of six for 62.5 
$\mathrm{ms}$ and there was $62.5 \mathrm{~ms}$ between each flash. During calibration, 120 flashes were used for each item (10 targets). Written Symbol Rate (Furdea, 2009) optimized the number of flashes used for the online copy task.

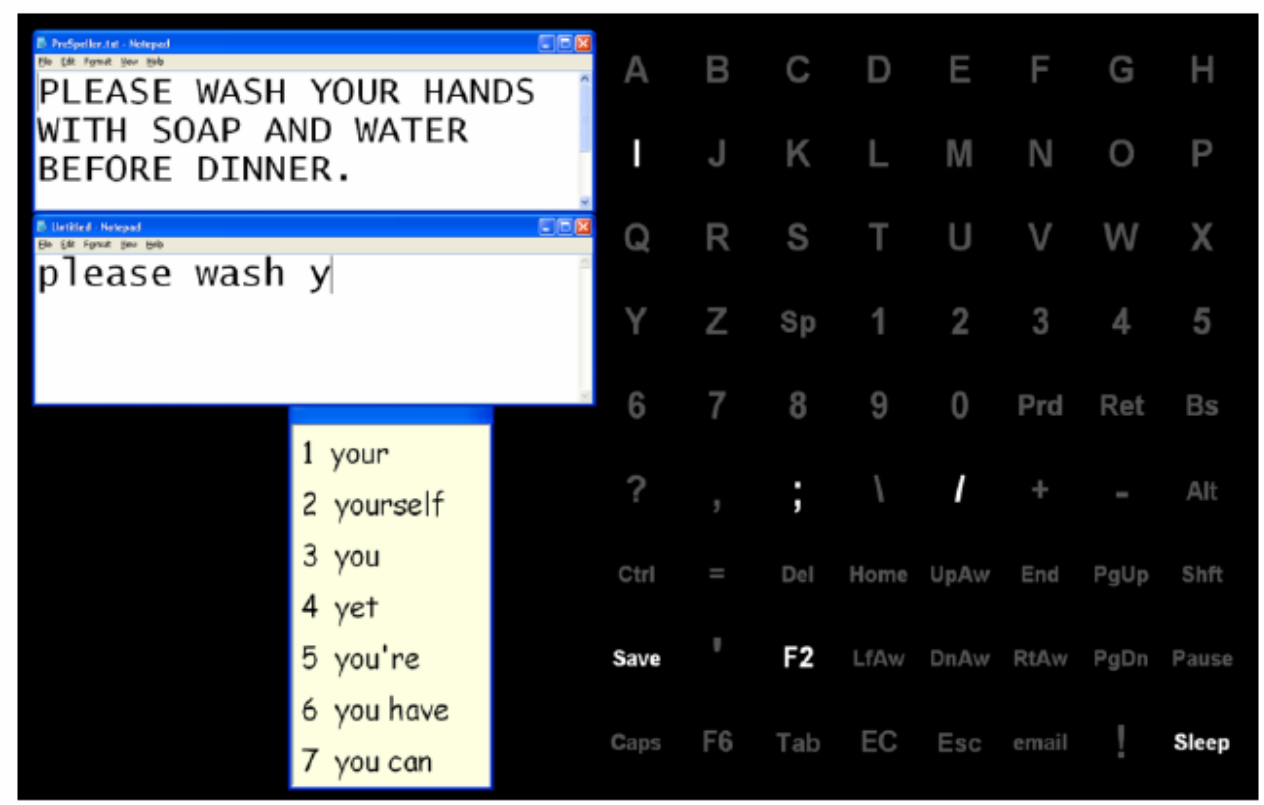

Fig. 12. The $8 \times 9$ matrix and additional windows used during the online spelling phase of the experiment. Right: the flashing matrix used to make item selections. Left top: the sentence target window. Left middle: the sentence output window. Left bottom: the predictive spelling window used in the predictive speller condition

During the copy task, a Notepad window (target window) adjacent to the matrix showed the sentence to copy (Fig.12). Selections were made by attending to the matrix (Fig.12 right) and counting how many times the desired item flashed. Output was presented in a second Notepad window (output window, Fig.12 middle left) that was located directly below the target window (Fig.12 top left). In the condition without the predictive speller, subjects selected items, evaluated output, and determined what item to choose next; the next item or Backspace. In the predictive speller condition, the predictive speller application program window was directly below the output window. After each selection, the predictive speller program would populate a numbered list of seven words. Subjects evaluated feedback in the predictive speller program window to determine if the desired word was listed; if so, the subject attended to the number in the matrix corresponding to the desired word on the next selection; when a number is selected the predictive speller program sends a word and space to the output window. If an incorrect number is selected, the participant can select Escape from matrix on the next selection, which returns the output window to its prior state, thus, eliminating multiple backspaces.

Table 4 (columns 1 and 2) shows that the non-predictive speller condition provided significantly higher accuracy than the predictive speller condition, $90 \%$ and $85 \%$, respectively $(t(23)=2.15, p=0.04, d=0.40)$. 


\begin{tabular}{|c|c|c|c|c|c|c|}
\hline Subject & $\begin{array}{l}\text { Predictive } \\
\text { Accuracy }\end{array}$ & $\begin{array}{c}\text { Non-predictive } \\
\text { Accuracy }\end{array}$ & $\begin{array}{c}\text { Predictive } \\
\text { Bit Rate } \\
\end{array}$ & $\begin{array}{c}\text { Non-predictive } \\
\text { Bit Rate }\end{array}$ & $\begin{array}{c}\text { Predictive } \\
\text { Theoretical BR }\end{array}$ & $\begin{array}{l}\text { NonPredictive } \\
\text { Theoretical BR }\end{array}$ \\
\hline 1 & 96.88 & 95.31 & 23.70 & 28.26 & 39.33 & 56.09 \\
\hline 2 & 88.89 & 87.50 & 19.93 & 19.54 & 32.62 & 32.38 \\
\hline 3 & 70.00 & 88.16 & 11.48 & 16.46 & 17.11 & 24.58 \\
\hline 4 & 79.59 & 89.86 & 18.78 & 20.41 & 33.52 & 33.82 \\
\hline 5 & 91.89 & 92.65 & 17.71 & 15.39 & 26.33 & 21.50 \\
\hline 6 & 87.18 & 95.31 & 21.73 & 22.58 & 38.70 & 37.39 \\
\hline 7 & 91.67 & 100.00 & 21.21 & 24.85 & 34.96 & 41.13 \\
\hline 8 & 81.13 & 87.50 & 15.79 & 21.72 & 24.66 & 38.86 \\
\hline 9 & 80.95 & 70.83 & 17.35 & 17.60 & 28.64 & 35.05 \\
\hline 10 & 82.35 & 98.33 & 22.28 & 29.98 & 44.12 & 59.45 \\
\hline 11 & 80.00 & 91.18 & 12.11 & 14.91 & 16.87 & 20.79 \\
\hline 12 & 77.59 & 82.50 & 11.55 & 12.69 & 16.10 & 17.70 \\
\hline 13 & 82.22 & 93.94 & 17.61 & 22.00 & 28.91 & 36.45 \\
\hline 14 & 94.29 & 77.17 & 22.01 & 14.57 & 36.00 & 22.81 \\
\hline 15 & 91.18 & 95.31 & 19.10 & 20.51 & 29.70 & 32.05 \\
\hline 16 & 94.29 & 85.25 & 18.52 & 15.62 & 27.51 & 23.29 \\
\hline 17 & 72.50 & 77.23 & 8.18 & 11.45 & 10.48 & 15.98 \\
\hline 18 & 91.89 & 100.00 & 26.69 & 31.12 & 52.65 & 61.70 \\
\hline 19 & 100.00 & 100.00 & 25.00 & 24.85 & 41.13 & 41.13 \\
\hline 20 & 96.88 & 91.18 & 21.25 & 19.00 & 33.01 & 29.70 \\
\hline 21 & 86.67 & 91.43 & 16.06 & 14.95 & 23.92 & 20.82 \\
\hline 22 & 57.58 & 83.67 & 5.02 & 15.13 & 6.19 & 22.62 \\
\hline 23 & 67.07 & 96.77 & 11.80 & 16.55 & 18.46 & 23.06 \\
\hline 24 & 94.44 & 84.15 & 20.27 & 15.28 & 31.54 & 22.82 \\
\hline Average & 84.88 & 89.80 & 17.71 & 19.39 & 28.85 & 32.13 \\
\hline Stand. Dev. & 10.59 & 7.78 & 5.38 & 5.39 & 10.95 & 12.83 \\
\hline Stand. Error & 2.16 & 1.59 & 1.10 & 1.10 & 2.24 & 2.62 \\
\hline
\end{tabular}

Table 4. Online test phase accuracy, bit rate, and theoretical bit rate for the predictive speller and non-predictive speller.

In contrast, output selections/min, 5.3, was significantly higher in the predictive speller condition than in the non-predictive speller condition, 3.8 selections/min $(\mathrm{t}(23)=6.05, \mathrm{p}<$ $.001, \mathrm{~d}=0.78$ ) (Table 5 columns 3 and 4). Moreover, the total time to complete the task was significantly less in the predictive speller condition, $12.4 \mathrm{~min}$, than in the non-predictive speller condition, 20.2min $(\mathrm{t}(23)=7.52, \mathrm{p}<.001, \mathrm{~d}=0.84)$ (Table 5 columns4 and 5). P300 amplitude at $\mathrm{Pz}$ was significantly higher in the non-predictive condition. Reduced amplitude in the predictive speller condition may be due to additional workload (Kramer, 1983). 


\begin{tabular}{|c|c|c|c|c|c|c|c|}
\hline Subject & $\begin{array}{c}\text { Predictive } \\
\text { Sets Per Seq. }\end{array}$ & $\begin{array}{l}\text { NonPredictive } \\
\text { Sets Per Seq. }\end{array}$ & $\begin{array}{c}\text { Predictive } \\
\text { Completion (min) }\end{array}$ & $\begin{array}{c}\text { NonPredictive } \\
\text { Completion (min) }\end{array}$ & $\begin{array}{l}\text { Predictive } \\
\text { Sel (min) }\end{array}$ & $\begin{array}{l}\text { NonPredictive } \\
\text { Sel (min) }\end{array}$ & $\begin{array}{c}\text { Output } \\
\text { Chars (min) }\end{array}$ \\
\hline 1 & 3.00 & 2.00 & 7.80 & 12.70 & 4.10 & 5.04 & 7.44 \\
\hline 3 & 4.00 & 4.00 & 24.00 & 22.70 & 3.33 & 3.35 & 2.42 \\
\hline 4 & 2.50 & 3.00 & 10.92 & 17.15 & 4.49 & 4.02 & 5.31 \\
\hline 5 & 4.00 & 5.00 & 11.00 & 23.58 & 3.36 & 2.88 & 5.27 \\
\hline 7 & 3.00 & 3.00 & 8.90 & 14.40 & 4.04 & 4.03 & 6.52 \\
\hline 8 & 3.50 & 2.50 & 14.47 & 16.10 & 3.66 & 4.47 & 4.01 \\
\hline 9 & 3.00 & 2.00 & 10.40 & 23.90 & 4.04 & 5.02 & 5.58 \\
\hline 10 & 2.00 & 2.00 & 10.10 & 11.90 & 5.05 & 5.04 & 5.74 \\
\hline 11 & 5.00 & 5.00 & 19.15 & 23.70 & 2.87 & 2.87 & 3.03 \\
\hline 15 & 3.50 & 3.50 & 9.25 & 17.50 & 3.68 & 3.66 & 6.27 \\
\hline 16 & 4.00 & 4.00 & 10.40 & 18.20 & 3.37 & 3.35 & 5.58 \\
\hline 17 & 5.00 & 5.00 & 17.75 & 35.25 & 2.25 & 2.87 & 3.27 \\
\hline 18 & 2.00 & 2.00 & 7.30 & 11.50 & 5.07 & 5.04 & 7.95 \\
\hline 19 & 3.00 & 3.00 & 7.65 & 14.40 & 4.05 & 4.03 & 7.58 \\
\hline 20 & 3.50 & 3.50 & 8.70 & 18.60 & 3.68 & 3.66 & 6.67 \\
\hline 21 & 4.00 & 5.00 & 13.40 & 24.45 & 3.36 & 2.86 & 4.33 \\
\hline 22 & 3.50 & 4.00 & 16.95 & 29.30 & 1.95 & 3.34 & 3.42 \\
\hline 23 & 3.50 & 5.00 & 22.45 & 21.60 & 3.65 & 2.87 & 2.58 \\
\hline 24 & 3.50 & 4.00 & 9.80 & 24.50 & 3.67 & 3.35 & 5.92 \\
\hline
\end{tabular}

Table 5. Online test phase sets per sequence, time to complete the sentence, and selections per minute in the predictive speller and non-predictive speller paradigms, and the predictive output in characters per minute.

Accuracy was lower in the predictive speller condition than in the non-predictive speller condition. Nonetheless, the predictive speller saved 7.4min as compared to the same overall output in the non-predictive speller. Over a period of one hour this translates to 92 extra selections. Accuracy in the predictive speller was $85 \%$; it is unclear if similar savings are possible with lower accuracy. These results suggest that a predictive speller can dramatically improve P300-BCI performance.

Support: NIH/NIBIB \& NINDS (EB00856); NIH/NIDCD (R21 DC010470-01); NIDCD, NIH (1 R15 DC011002-01).

\section{Project 8: Innovations in P300-based BCI presentation methods}

George Townsend

Since its original inception by Farwell and Donchin in 1988, the P300-based interface has always flashed in rows and columns. Disassociating the physical rows and columns of the target matrix from the way targets are grouped to flash in the P300 interface brings about a number of advantages. Supported by the Wadsworth BCI group (Wolpaw, 2003), the Algoma University BCI Laboratory introduced the "checkerboard" paradigm in which targets are grouped in rows and columns in two "virtual matrices" taken from the white and from the black squares of a checkerboard that is overlaid on the physical matrix (see Figure 13). 
A) WADSWORTH (W)

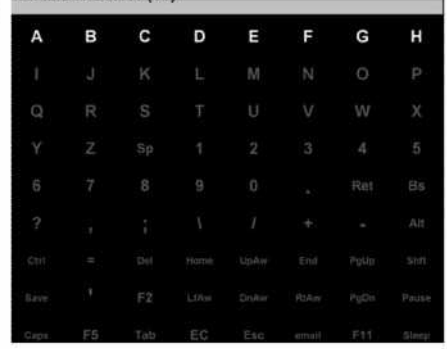

B)

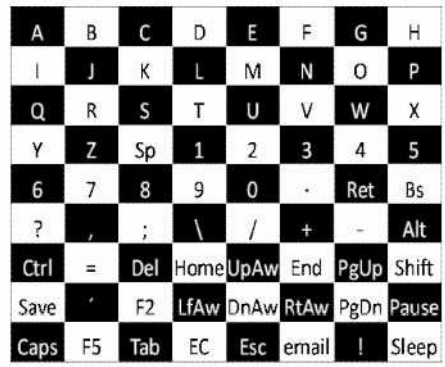

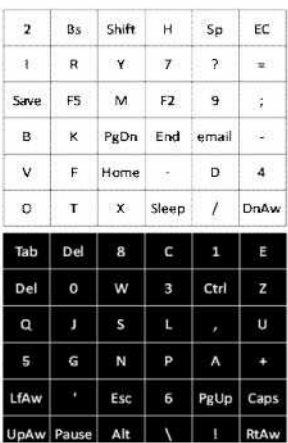

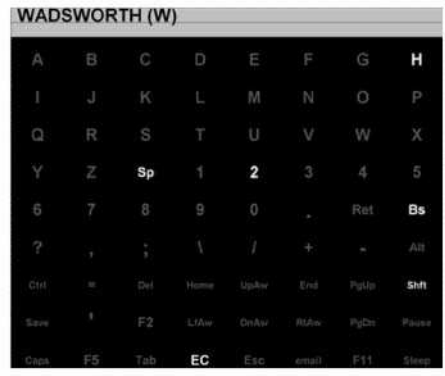

Fig. 13. A: The Row-Column paradigm (RCP) for the $8 \times 9$ matrix, with one row flashing. $\mathrm{B}$ : The Checkerboard paradigm (RCP) for the $8 \times 9$ matrix. On the left is the checkerboard pattern. In the middle are the two virtual $6 \times 6$ matrices derived from the checkerboard. On the right is the matrix as presented to the participant with the top row of the white matrix flashing.

This innovative approach eliminated the troublesome "double flash" problem and mitigated the "adjacency distraction" problem that plagued the original P300 implementation. Subsequent studies have shown this version to provide an increase in both speed and accuracy over the traditional implementation. The approach has been studied online in real time with both able-bodied subjects as well as disabled individuals.

Perhaps most important is the success that this new approach has had with those who suffer from ALS. The "checkerboard" interface was featured on CBS's primetime news program "60 minutes," where it was demoed by both the commentator as well as the ALS patient Scott Mackler. http://www.cbsnews.com/video/watch/?id=5228109n\&tag=related; photovideo. The flashing pattern used in the demonstration was the one developed by the Algoma University Lab. There was a dramatic improvement experienced by the ALS patients tested on the new interface in a preliminary study.

These improvements are only the beginning of what might be possible. The disassociation of the "flash groups" from the physical matrix is now being taken further and the flash groups become purely "abstract" bearing no relationship to rows or columns either physical or virtual. Our experience with the "checkerboard" has brought us to realize that performancebased constraints rather than physical constraints should be used to guide the organization of flash groups in the P300-based BCI. Once the shackles of physical constraints are cast off, we realize that there are $C(n, k)$ ways in which to flash $k$ target flashes in amongst $n$ total 
flashes per sequence, or $\mathrm{n} ! /(\mathrm{k} !(\mathrm{n}-\mathrm{k}) !)$. In a sequence of 36 flashes in which each target flashes five times, there are 376,992 ways in which this can be accomplished. In the case of a target matrix in which there are only 72 items, this leads to a high degree of flexibility allowing for imposing many constraints designed to improve the performance of the interface. This includes those already addressed by the original checkerboard design as well as many others such as minimizing the number of flashes that a target has in common with other targets. We are currently working on new paradigms based on these ideas.

As this research begins to push the limits of the P300 interface, issues with the timing limitations associated with computer video monitors have begun to surface and we have developed a self contained LED based display compatible with the BCI2000 platform to address these issues. This new specialized hardware is currently under testing and shows promising results when used in conjunction with these new paradigms based on performance guided flash organizations. Participants involved in the studies have expressed a preference for the new paradigms and the new LED based display demonstrating a clear improvement in the usability of the interface.

\section{Project 9: Operant conditioning to identify independent, volitionally-controllable patterns of neural activity}

Steven M. Chase, Andrew S. Whitford, Andrew B. Schwartz

One of the most exciting applications of brain-computer interface (BCI) devices is the restoration of hand and arm function to individuals who have lost that ability. This is also one of the most challenging applications: a human hand and arm have more than 20 independently controllable degrees of freedom (DoFs) that must be coordinated to achieve even simple tasks. To date, the most successful application of a BCI toward functional arm restoration has been the demonstration of a monkey using a 4 DoF robotic arm to feed itself (Velliste, 2008). While a remarkable achievement, this is still well below the number of controllable DoFs required to replace the capability of a lost limb.

One of the major difficulties in establishing high dimensional control is the problem of calibration: when recording from a network of sensors, how should the patterns of activity in the sensor array be mapped to the controllable degrees of freedom in the device? A number of different approaches have been used to solve this problem. One method is to perform the calibration on natural arm movements (Ganguly, 2009, Wessberg, 2000). This technique is clearly inappropriate in a clinical setting when the subject cannot move his natural arm. Another approach is to instruct the subject to produce imagined movements while recording the sensor activity (Velliste, 2008, Taylor, 2002, Hochberg, 2006, Schalk, 2008). While this technique has proven successful in many experimental settings, it relies on there being a clear representation of the imagined movement in the recorded sensors. If the sensors are recording neural activity that represents other movements or volitional signals than the instructed movement, this information will be missed. A third possibility is to use operant conditioning to discover the volitionally controlled signals that affect the recorded population. This technique, first performed on single neurons by Fetz (Fetz, 1969), has been tried with some success in low dimensional BCI devices (Moritz, 2008, Birbaumer, 1999). However, without modification this technique cannot be extended to the control of high numbers of dimensions, for the following reasons. First, mapping a single neuron or sensor to a single DoF can be noisy; a preferable approach would reduce noise by averaging across multiple neurons or sensors. Second, Fetz' approach cannot constrain multiple neurons to be 
mutually uncorrelated, and so cannot be extended to gain multiple dimensions of control. The technique we propose here allows us to (1) find a pattern of correlated sensor or neural activity that can be used to control a single DoF, and (2) find multiple patterns of such activity that are mutually uncorrelated. Furthermore, because the technique relies on biofeedback, it need not be assumed that the recorded neural activity represents a particular movement; in principle, any latent volitionally controllable signal that affects the recorded activity can be uncovered.

The procedure for identifying orthogonal patterns of brain activity is as follows. The monkey sits in a primate chair facing a monitor that displays two concentric rings: a blue target ring and a green feedback ring. The radius $r$ of the feedback ring is controlled by the subject's neural activity, through the equation $r=a f{ }^{*} \mathbf{w}$. Here, $\mathbf{f}=[\mathrm{f} 1, \ldots, \mathrm{fn}]$ is the vector containing the sampled firing rate from $\mathrm{n}$ neurons (or equivalently, activity from $\mathrm{n}$ sensors), $\mathbf{w}=[\mathrm{w} 1, \ldots \mathrm{wn}]^{\mathrm{T}}$ is the weight vector that determines how each neuron contributes to the radius, and a is a normalizing constant. The goal of the task is for the subject to control his neural activity such that the feedback ring hits the target ring. After hitting two target rings (an outer ring and an inner ring) consecutively, within a timeout period, a reward is given. We start with the standard Fetz task (Fetz, 1969), where we use the firing rate of only one neuron to control the radius of the feedback ring. We've found that the subject can learn, by trial and error, to achieve volitional control over approximately $>50 \%$ of the recorded neurons within $\sim 2$ minutes, at least for neurons with sufficiently high baseline rates (Fig. $14 \mathrm{~A})$. Once volitional control has been established with one neuron, we pick another and use it to drive the ring. This procedure continues for a small sample of cells, typically between 2 and 10, taking between 5 and 20 minutes. During single-unit control there is significant correlation in the population response, even though the other units do not contribute to control. This suggests that if we were to average over the population appropriately, we might uncover cleaner, less noisy control signals. We extract the first pattern of neural activity by performing a principal components analysis (PCA) on the neural data. Specifically, we create a data matrix $\mathrm{F}$ that contains all of the firing rate samples from the successful trials so far observed $\left(F=\left[f_{1} T, \ldots, f_{m}^{T}\right]\right.$, where $m$ is the number of successful trials). We then perform PCA on this data matrix to find the single vector that explains the most variance in the data. Mathematically, we solve $\mathbf{w}_{\mathrm{PC} 1}=\operatorname{argmax}_{\mathrm{w}}\left\{\operatorname{Var}\left(\mathrm{w}^{\mathrm{T}} \mathrm{F}\right)\right\}$, subject to the constraint that || $\mathbf{w}||=1$. We then use this vector to control the feedback ring. Control with the first PC is typically very good (Fig. 14B); noisiness that can result when sampling a single neuron is reduced when projecting the firing rates from the entire population onto the first PC. To find the next orthogonal pattern of controllable activity, we combine all of the data we have taken to this point (both data from when single neurons were in control and from when the first PC was used for control) into a data matrix $F_{\text {total }}$. We then project this data into the space orthogonal to the first $\mathrm{PC}$, through the equation $\mathrm{F}=\mathrm{F}_{\text {total }}-\mathbf{w}_{\mathrm{PC} 1} \mathbf{W}_{\mathrm{PC}}{ }^{\mathrm{T}} \mathrm{F}_{\text {total }}$. Essentially, we take every vector of firing rates we've observed and subtract off the component that lies along $\mathbf{w}_{\mathrm{PC} 1}$. We then again perform PCA, to find the single vector that explains the maximum amount of variance in $\mathrm{F} \perp$. By construction, this vector is guaranteed to be orthogonal to $\mathbf{w}_{\mathrm{PC1}}$. We then apply this vector as the weight vector that controls the feedback ring. This procedure can be iterated until the subject can no longer control the ring, or until there are as many components as there are neurons. We find that with recordings consisting of only 30 neurons, we can reliably find $\sim 5$ orthogonal components that can be volitionally controlled (Fig. 14C). 


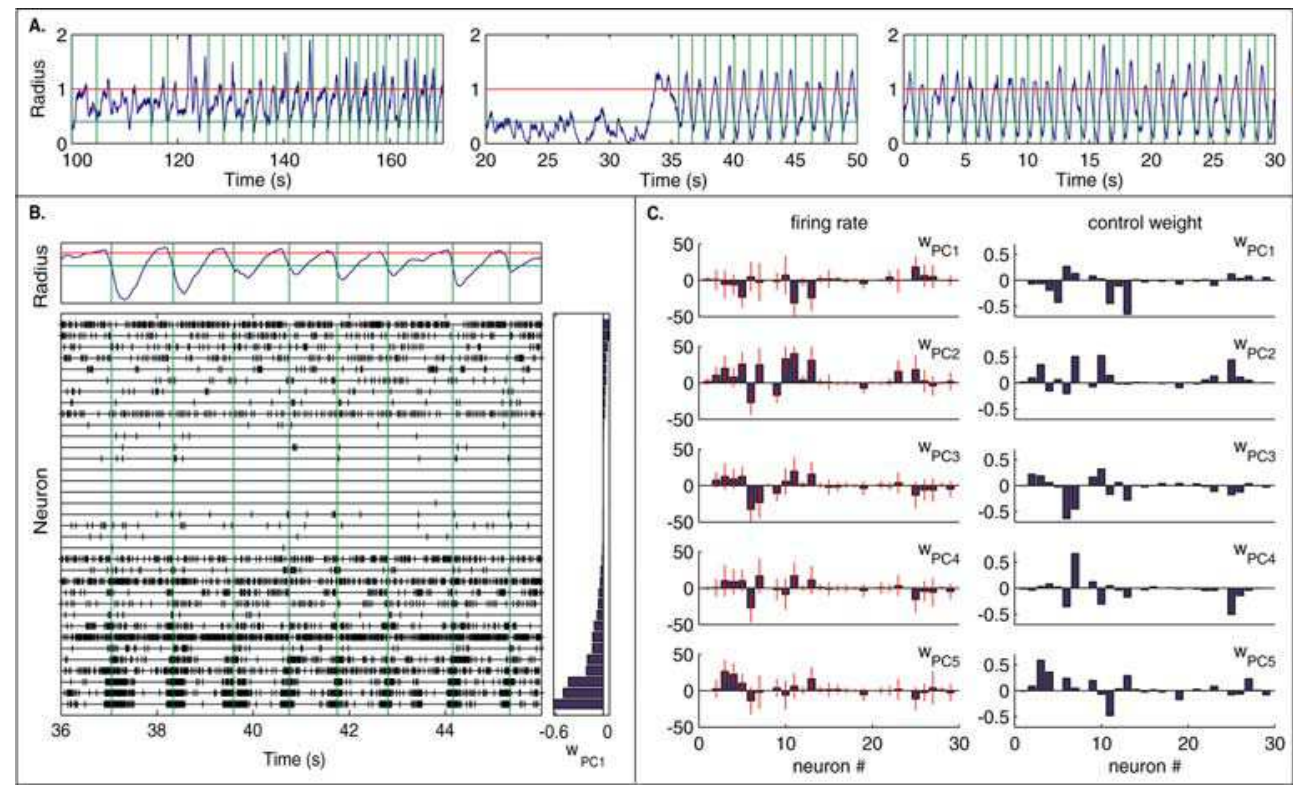

Fig. 14. Patterns of neural activity revealed through operant conditioning. A. Three examples of control with single neurons. The plots show the radius of the feedback ring as a function of time since the neuron was put in control of the ring. The first two plots show examples of the subject learning, through trial and error, volitional control of the ring. The third shows an example where the control was immediate, because the control neuron was correlated with the neuron previously in control. Red and green horizontal lines denote outer and inner target ring positions, respectively; green vertical lines denote successes. B. Raster plot during control with $\mathrm{wPC}$. Units are sorted according to their contribution to the $\mathrm{PC}$, shown on the right. $\mathrm{C}$. The left column displays histograms of the difference in firing rate between the outer and inner targets for all neurons; each plot shows control with a different PC. Red bars display +/- 1 SD. The corresponding PC weights are shown on the right.

Often, the biggest problem with achieving high dimensional control with a BCI device is training. The training procedure has two components: first, a mapping between the neural activity and the control of each device DoF must be established; second, given a particular mapping, the subject must learn how to shape his neural activity to achieve the desired movement. In our experience, subjects have little ability to control a device when an arbitrary mapping is applied between the neural activity and the device (data not shown). On the other hand, humans have little trouble learning to control a computer cursor with a cyber glove, even when the joint angles in the glove are arbitrarily mapped to cursor movements (Liu, 2008, Mosier, 2005). The difference is that the cyber glove maps independent volitional signals to cursor movements, while arbitrary mappings of the neural activity do not preserve the independence of the volitional signals. Using our procedure, the underlying latent volitional signals can be recovered and mapped to particular device DoFs while maintaining their independence. In addition to reduced training times and a consistent framework in which to calibrate the operation of multiple BCI devices, the 
procedure we have developed has a number of basic science applications. In particular, it allows us to explore the fundamental limits on learning and adaptation, by probing a subject's ability to sculpt the correlations in a network of neurons. Ultimately, using models of the volitional control signals and the functional connectivity of the network, we hope to predict the behavior of the network in response to different behavioral challenges.

\section{Project 10: Neurorehabilitation for chronic-phase stroke using a brain-machine interface}

Kimiko Kawashima, Keiichiro Shindo, Junichi Ushiba, Meigen Liu

Spelling devices or robotic-arm control with BCIs have been widely developed for the purpose to substitute lost motor function in patients with spinal cord injury and neuromuscular diseases. In addition to such 'functional compensation with $\mathrm{BCI}^{\prime}$, rather a new concept of 'neurorehabilitation with $\mathrm{BCI}^{\prime}$, in facilitation of neural sensory-motor activity using volitionally controlled motor-driven orthosis, might also be valuable in rehabilitation.

To test the feasibility of the concept of BCI neurorehabilitation, we recruited two patients with hemiplegic stroke due to sub-cortical lesions (Patient A (PAT-A): corona radiata infarction, Patient B (PAT-B): putaminal hemorrhage) for this study, which was approved by the local ethics committee, and the patients gave informed consent. The scores of Stroke Impairment Assessment Set (SIAS) finger function test were 1A in both patients, meaning no observable volitional finger movement. Spasticity was present in fingers and wrist flexors, and paralyzed fingers and arms were flexed and supinated in a typical Wernicke-Mann posture. More than one year had passed since the stroke, and thus further functional recovery was not expected.

Our BCI was designed to activate a motor-driven orthosis that was attached to the paretic hand in response to the motor intention of the patient's hand (Figure 15a). Using $\mathrm{Ag} / \mathrm{AgCl}$ scalp electrodes $(\varphi=10 \mathrm{~mm})$, the EEG was recorded over the sensorimotor cortex of both hemispheres (C3 and C4, with four neighbor Laplacian) and digitized at 256 $\mathrm{Hz}$ using an EEG amplifier (g.tec Guger Technologies, Graz, Austria). The amplitude of the event-related desynchronization (ERD) within 8-35 Hz was calculated every $300 \mathrm{~ms}$ with a time-sliding window of $1 \mathrm{~s}$, as a feature that represents the participant's motor intention [4]. The magnitude of ERD in both hemispheres was classified with linear discriminant analysis to judge whether the patient was at rest or was intending hand opening. The orthosis was triggered to move after a motor intention of 2-5 s (which was set depending on patient's proficiency), if the accuracy of the EEG classification exceeded $50 \%$ (Fig.15b). This protocol was repeated for 1 hour once or twice a week over a period of 4 to 7 months.

An evaluation of the BCI neurorehabilitation demonstrated an enhancement of ERD with motor imagery. Comparison of the results of pre- and post-BCI training revealed that the ERD values significantly decreased over both hemispheres (Fig. 15c), and was more prominent in ipsi-lesional side. Enhancement of ERD resulted in a higher accuracy of BCI (Patient A: 38\% -> 97\%, Patient B: 55\% -> 63\%). Surface electromyography (EMG) recorded from finger extensors (extensor digitorumcommunis) showed improvement of volitional changes in amplitude (Fig.15d). Reappearance of EMG with a long-term use of BCI is outstanding because previous research found changes of cortical activity only [Daly \&Wolpaw 2008].

Also, qualitatively the results were very positive; enthusiastic comments from the patients suggested that they had experienced raised awareness of the paretic hand. This should 
stimulate them to use their paretic hand in their daily activities. In addition, the increase in the EMG suggests the possible use of other therapeutic methods such as EMG-triggered electrical stimulation, in which minimal voluntary muscle control is needed, for further rehabilitation.

BCI training may have induced EEG changes over the sensorimotor cortices, thereby improving muscle control and increasing the efficiency of rehabilitation. In the future, BCI technology might be a promising tool to restore more effective motor control in patients with stroke.

This study was partially supported by the Strategic Research Program for Brain Sciences (SRPBS) from the Ministry of Education, Culture, Sports, Science and Technology, Japan.
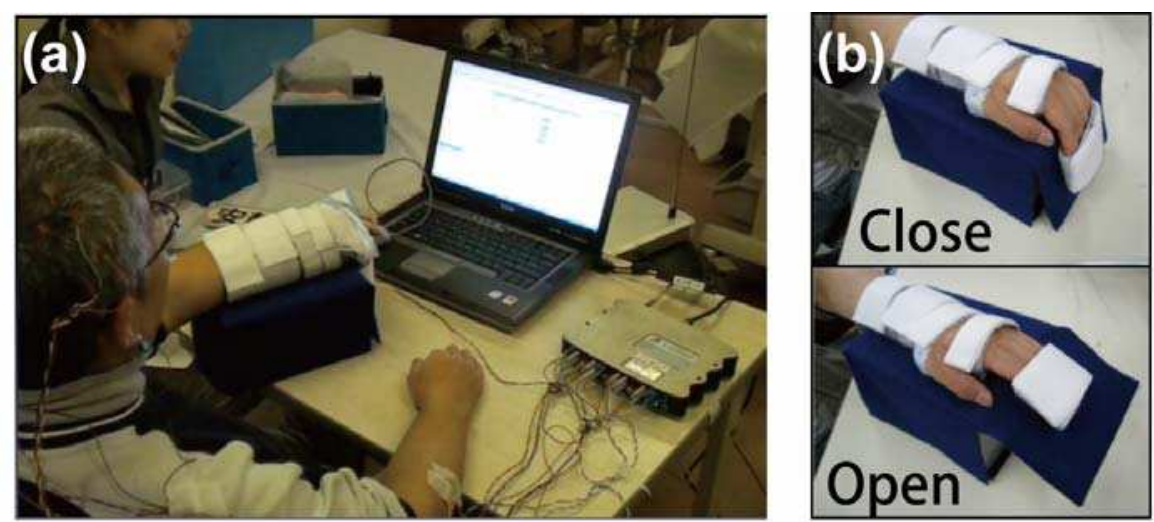

(c)

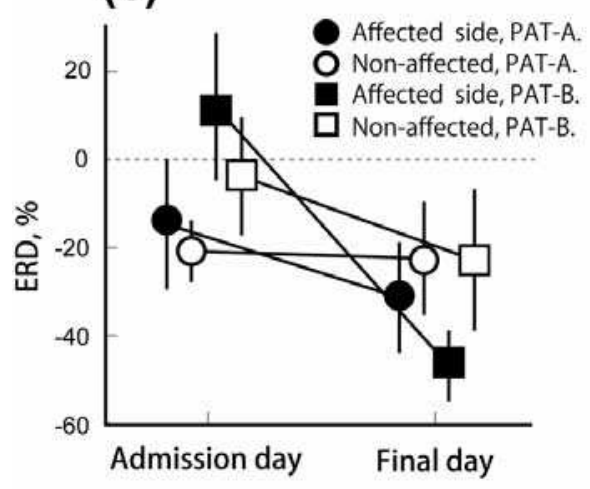

(d)

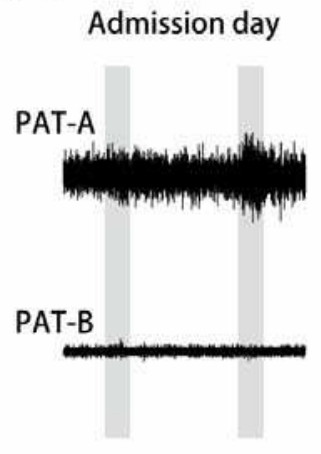

Final day

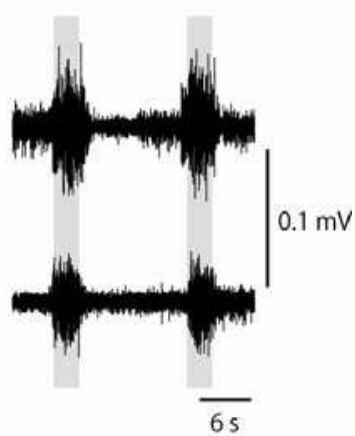

Fig. 15. Experimental setup and changes of ERD and EMG by BCI neurorehabilitation. (A) Overview of the experiment. (B) Action of the motor-driven hand orthosis. (C) ERD changes by BCI neurorehabilitation. Bar indicates standard deviation. (D) EMG changes by $\mathrm{BCI}$ neurorehabilitation. Shaded period indicates when patients were intending finger extension. 


\section{Discussion}

Out of 57 high quality submissions, the jury nominated the 10 top-ranked candidates for the BCI Research Award in April 2010. The jury then selected the winner of the 2010 BCI Award at the BCI 2010 conference in Monterey, California, in June 2010. The winning team was Cuntai Guan, Kai Keng Ang, Kok Soon Phua, Chuanchu Wang, Zheng Yang Chin, Haihong Zhang, Rongsheng Lin, Karen Sui Geok Chua, Christopher Kuah, Beng Ti Ang (A*STAR, Singapore), and their project was "Motor imagery-based Brain-Computer Interface robotic rehabilitation for stroke". This project represents a study with 26 subjects that combines current understanding of neurophysiology, rehabilitation, computer science, and signal processing to realize one of the most impressive studies in the rapidly growing area of brain-computer interfacing for stroke rehabilitation.

Table 5 shows a categorization of the BCI Award 2010 nominees into utilized control signals and application areas. The majority of 8 projects used EEG as input signal and 6 utilized the P300/N200 response. This has several reasons: (i) the EEG P300 response is easy to measure and a non-invasive method, (ii) it requires just a few minutes of training, (iii) works with the majority of subjects and (iv) gives a goal-oriented control signal that is especially suited for spelling and control application where no continuous control signal is needed (e.g., Internet surfing, painting). Actually, all the spelling/Internet/art applications were controlled with the N200/P300 strategy. Two projects used motor imagery (MI) in order to generate a continuous control signal. Both MI projects used the BCI system for the activation of the sensori-motor cortex for stroke rehabilitation that cannot be done with N200/P300- or SSVEP-based BCI systems. No SSVEP-based BCI systems were nominated for the BCI Award. This is surprising, because SSVEP-based systems achieve high accuracies and information transfer rate and can be operated by the majority of people. The reason could be that for goal-oriented control, the P300 principle is better suited because it gives more options by using standard computer screens. SSVEP-based systems required LED stimulators but can also use computer screens. Especially in the latter case, it is complicated to realize a high number of different frequencies. But it becomes more difficult for a high number of LEDs compared to arranging 50-100 icons on the screen for a P300 speller.

One fMRI- and one spike-based project were nominated. fMRI-based BCIs are more complicated to operate but have the big advantage of the good spatial resolution which allows to read out different control signals compared to EEG-based systems. Instead of selecting single characters, fMRIs can be used to extract, e.g., the semantic output code to form words and sentences, to play tennis, or to navigate in your home (Owen, 2008, Palatucci, 2009). Action potentials give the highest spatial and temporal resolution, but are require implantation of electrodes within the cortex. Nevertheless, spikes allow a very accurate control of BCI systems and can even be used for robotic control with high accuracy [Velliste, 2008].

Table 6 lists different properties of all the 57 projects submitted to the BCI Award 2010. Of particular interest is the high percentage of real-time BCI implementations that exist nowadays. Motor imagery is still the mostly used strategy to control a $\mathrm{BCI}$, followed by P300 and SSVEP. It is also not surprising that mostly EEG-based BCI systems are used because they are easier to handle and are cheaper. The mostly implemented application is spelling, ahead of general control (the papers did not mention a certain application) and stroke rehabilitation, wheelchair/robot or Internet control. $12.3 \%$ of the submission introduced a BCI platform or certain improvements of technology. 


\begin{tabular}{|c|c|c|c|c|c|c|c|c|}
\hline \multirow[t]{2}{*}{ Title } & \multicolumn{5}{|c|}{ Control signal } & \multicolumn{3}{|c|}{ Application } \\
\hline & fMRI & Spikes & N200/P300 & SSVEP & MI & Stroke & $\begin{array}{c}\text { Spelling/ } \\
\text { internet/art }\end{array}$ & $\begin{array}{c}\text { Algorithm } \\
\text { development }\end{array}$ \\
\hline $\begin{array}{l}\text { A high speed word } \\
\text { spelling BCI system } \\
\text { based on code } \\
\text { modulated visual } \\
\text { evoked potentials }\end{array}$ & & & $x$ & & & & $X$ & \\
\hline $\begin{array}{l}\text { Motor imagery-based } \\
\text { Brain-Computer } \\
\text { Interface robotic } \\
\text { rehabilitation for } \\
\text { stroke }\end{array}$ & & & & & $\mathrm{X}$ & $X$ & & \\
\hline $\begin{array}{l}\text { An active auditory } \\
\text { BCI for intention } \\
\text { expression in locked- } \\
\text { in }\end{array}$ & & & $X$ & & & & $x$ & \\
\hline \begin{tabular}{|l|} 
Brain-actuated Google \\
search by using \\
motion onset VEP
\end{tabular} & & & $x$ & & & & $x$ & \\
\hline $\begin{array}{l}\text { Brain Painting - "Paint } \\
\text { your way out" }\end{array}$ & & & $X$ & & & & $\mathrm{X}$ & \\
\hline $\begin{array}{l}\text { Thought Recognition } \\
\text { with Semantic Output } \\
\text { Codes }\end{array}$ & $X$ & & & & & & $X$ & \\
\hline $\begin{array}{l}\text { Predictive Spelling } \\
\text { with a P300-based } \\
\text { BCI: Increasing } \\
\text { Communication Rate }\end{array}$ & & & $x$ & & & & $X$ & \\
\hline $\begin{array}{l}\text { Innovations in P300- } \\
\text { based BCI Stimulus } \\
\text { Presentation Methods }\end{array}$ & & & $x$ & & & & $x$ & \\
\hline $\begin{array}{l}\text { Operant conditioning } \\
\text { to identify } \\
\text { independent, } \\
\text { volitionally- } \\
\text { controllable patterns } \\
\text { of neural activity } \\
\end{array}$ & & $X$ & & & & & & $x$ \\
\hline $\begin{array}{l}\text { Neurorehabilitation } \\
\text { for Chronic-Phase } \\
\text { Stroke using a Brain- } \\
\text { Machine Interface } \\
\end{array}$ & & & & & $\mathrm{X}$ & $x$ & & \\
\hline Total & 1 & 1 & 6 & & 2 & 2 & 7 & 1 \\
\hline
\end{tabular}

Table 5. Categorization of the BCI Award nominees. 


\begin{tabular}{|c|c|c|c|}
\hline Property & Percentage (N=57) & Property & Percentage (N=57) \\
\hline Real-time BCI & 65.2 & Stroke & 7.0 \\
\hline Off-line algorithms & 17.5 & Spelling & 19.3 \\
\hline P300 & 29.8 & Wheelchair/Robot & 7.0 \\
\hline SSVEP & 8.9 & Internet/VR & 8.8 \\
\hline Motor imagery & 40.4 & Control & 17.5 \\
\hline EEG & 75.4 & Platform/Technology & 12.3 \\
\hline fMRI & 3.5 & & \\
\hline ECoG & 3.5 & & \\
\hline NIRS & 1.8 & & \\
\hline
\end{tabular}

Table 6. Properties of the submissions to the BCI Award 2010

\section{Conclusion}

The BCI Award 2010 was the first international Award for BCI system development. The submissions highlight the current status of BCI technology. It is important to identify the most promising technologies and application areas for a faster grow of the community. g.tec plans to continue the BCI Award on an annual basis. This should provide annual snapshots of the progress of $\mathrm{BCI}$ research and its exciting new applications.

\section{Acknowledgements}

We are grateful to the members of the jury of the BCI Award 2010: Gerwin Schalk, Theresa Vaughan, Eric Sellers, Dean Krusienski, Klaus-Robert Müller, Benjamin Blankertz, Bo Hong. We also thank Aysegul Gunduz and Peter Brunner for their assistance with the evaluation process. Finally, we thank Theresa Vaughan and Jon Wolpaw for organizing the BCI conference 2010 as platform for the BCI Award 2010. The work was funded by the EC projects Decoder, Brainable, Better.

\section{References}

Allison, B.Z., Wolpaw, E.W., Wolpaw, J.R. (2007). Brain-computer interface systems: progress and prospects. Expert Rev. Med. Devices 4(4). 463-474.

Ang, K.K., Chin, Z.Y., Zhang, H., and Guan, C. (2008). Filter Bank Common Spatial Pattern (FBCSP) in Brain-Computer Interface, in Proc. IJCNN'08, pp. 2391-2398.

Ang, K.K., Guan, C., Chua, K.S.G., Ang, B.T., Kuah, C.W.K., Wang, C., Phua, K.S., Chin, Z.Y., and Zhang, H. (2009). A clinical study of motor imagery-based braincomputer interface for upper limb robotic rehabilitation, in Proc. EMBC, pp. 59815984 .

Ang, K.K., Guan, C., Chua, K.S.G., Ang, B.T., Kuah, C.W.K., Wang, C., Phua, K.S., Chin, Z.Y., and Zhang, H. (2010). Clinical study of neurorehabilitation in stroke using EEG-based motor imagery brain-computer interface with robotic feedback", 32nd Annual International Conference of the IEEE Engineering in Medicine and Biology Society (EMBC), Buenos Aires, Argentina, 1- 4 September.

Beers, M.H., and Berkow, R. (2000). The Merck Manual of Geriatrics," 3rd ed New Jersey: Merck Research Laboratories. 
Bin, G., Gao, X., Yan, Z., Hong, B., \& Gao, S. (2009). An online multi-channel SSVEP-based brain-computer interface using a canonical correlation analysis method. Journal of Neural Engineering , 6pp.

Birbaumer N, Ghanayim N, Hinterberger T, Iversen I, Kotchoubey B, Kübler A, Perelmouter J, Taub E, Flor H. (1999). A spelling device for the paralysed. Nature, 398(6725), 297-8.

Birbaumer, N., Kubler, A., Ghanayim, N., Hinterberger, T., Perelmouter, J., Kaiser, J., Iversen, I., Kotchoubey, B., Neumann, N., Flor, H. (2000). The thought translation device (TTD) for completely paralyzed patients. IEEE Trans. Rehabil. Eng, 8(2): p. 190-193.

Birbaumer, N. (2006). Brain-computer-interface research: Coming of age, Clin. Neurophysiol., vol. 117(3), pp. 479-483.

Calautti C., and Baron, J.C. (2003). Functional Neuroimaging Studies of Motor Recovery After Stroke in Adults: A Review, Stroke, vol. 34(6), pp. 1553-1566.

Daly J.J. and Wolpaw, J.R. (2008). Brain-computer interfaces in neurological rehabilitation, The Lancet Neurology, vol. 7(11), pp. 1032-1043.

Farwell L.A., and Donchin, E. (1988). Talking off the top of your head: toward a mental prosthesis utilizing event-related brain potentials. Electroencephalogr. Clin. Neurophysiol., 70, 510-523.

Fetz EE. (1969). Operant conditioning of cortical unit activity. Science. 163(870), 955-8.

Furdea, A. et al. (2009). An auditory oddball (P300) spelling system for brain-computer interfaces. Psychophysiology 46, 617-625, doi:PSYP783 [pii] 10.1111/j.14698986.2008.00783.x.

Galán F, Nuttin M, Lew E, Ferrez PW, Vanacker G, Philips J, Millán J del R. (2008). A brainactuated wheelchair: asynchronous and non-invasive Brain-computer interfaces for continuous control of robots. Clin Neurophysiol. 119(9), pp.2159-69. Epub 2008 Jul 14.

Ganguly K, Carmena JM. (2009). Emergence of a stable cortical map for neuroprosthetic control. PLoS Biol, 7(7):e1000153. Epub 2009 Jul 21.

Guger, C. et al. (2009). How many people are able to control a P300-based brain-computer interface (BCI)? Neuroscience Letters, 462, 94-98, doi:S0304-3940(09)00819-2 [pii] 10.1016/j.neulet. 2009.06.045.

Hochberg LR, Serruya MD, Friehs GM, Mukand JA, Saleh M, Caplan AH, Branner A, Chen D, Penn RD, Donoghue JP. Neuronal ensemble control of prosthetic devices by a human with tetraplegia. Nature, 442(7099), 164-71.

Kramer, A. F., Wickens, C. D. \& Donchin, E. (1983).An analysis of the processing requirements of a complex perceptual-motor task. Hum Factors, 25, 597-621.

Krusienski, D.J., Sellers, E.W., Cabestaing, F., Bayoudh, S., McFarland, D.J., Vaughan, T.M., and Wolpaw, J.R. (2006).A comparison of classification techniques for the P300 Speller. J.NeuralEng, 3(4), 299-305.

Krusienski, D. J., Sellers, E. W., McFarland, D. J., Vaughan, T. M. \& Wolpaw, J. R. (2008). Toward enhanced P300 speller performance. J Neurosci Methods, 167, 15-21, doi:S0165-0270(07)00370-6 [pii] 10.1016/j.jneumeth. 2007.07.017.

Kübler, A.,Halder, S.,Furdea, A.,Hösle, A. (2008). Brain painting - BCI meets art, Proceedings of the 4th International Brain-Computer Interface Workshop and Training Course, 361-366. 
Leuthardt, E.C., Schalk, G., Wolpaw, J.R., Ojemann, J.G., and Moran, D.W. (2004). A braincomputer interface using electrocorticographic signals in humans. J Neural Eng. 1, pp.63-71.

Liu X, Scheidt RA. (2008). Contributions of online visual feedback to the learning and generalization of novel finger coordination patterns. J Neurophysiol, 99(5), pp.254657.

Martens, S. M., Hill, N. J., Farquhar, J., Scholkopf, B. (2009).Overlap and refractory effects in a brain-computer interface speller based on the visual P300 event-related potential. J Neural Eng 6, 026003, doi:S1741-2560(09)96900-2 [pii] 10.1088/17412560/6/2/026003.

Moritz CT, Perlmutter SI, Fetz EE. (2008). Direct control of paralysed muscles by cortical neurons. Nature, 456(7222), 639-42.

Mosier KM, Scheidt RA, Acosta S, Mussa-Ivaldi FA, (2005). Remapping hand movements in a novel geometrical environment. J Neurophysiol., 94(6), pp.4362-72.

Münßinger J, Halder S, Kleih SC, Furdea A, Raco V, Hoesle A and Kubler A (2010). Brain Painting: First Evaluation of a New Brain-Computer Interface Application with ALS-Patients and Healthy Volunteers. Front. Neurosci. doi: 10.3389/fnins.2010.00182

Münßinger, J.I., Halder, S., Kleih, S.C., Furdea, A., Raco, V., Hösle, A., Kübler, A. (in revision). Brainpainting: Evaluation of a newbrain-computer interface application with ALS-patients and healthy volunteers, Frontiers in Neuroprosthetics.

Nijboer, F., Sellers, E., Mellinger, J., Matuz, T., Furdea, A., Halder, S., Mochty, U., Jordan, M.,Krusienski, D.,Wolpaw, J.R., Birbaumer, N., Kübler, A. (2008). A P300-based brain-computer interface for people with amyotrophic lateral sclerosis, Clinical Neurophysiology, pp. 1909-1916.

Owen, A.M., Coleman, M.R. (2008). Using neuroimaging to detect awareness in disorders of consciousness. Funct Neurol. 23(4), 189-94.

Palatucci, M., et al. (2009).Zero-shot learning with semantic output codes. Neural Information Processing Systems. NIPS.

Pfurtscheller G, Bauernfeind G, Wriessnegger SC, Neuper C. (2010) Focal frontal (de)oxyhemoglobinresponses during simple arithmetic. Int J Psychophysiol, 76(3), pp.186-92.

Pfurtscheller, G., Allison, B.Z., Brunner, C., Bauernfeind, G., Solis-Escalante, T., Scherer, R., Zander, T.O., Mueller-Putz, G., Neuper, C., Birbaumer, N. (2010). The Hybrid BCI. Front Neurosci, 4, 42.

Piccione, F., Giorgi, F., Tonin, P., Priftis, K., Giove, S., Silvoni, S., Palmas, G., Beverina, F. (2006). P300-based brain computer interface: Reliability and performance in healthy paralyzed participants, Clinical Neurophysiology, pp. 531-537.

Townsend, G. T. et al. (2010). A novel P300-based brain-computer interface stimulus presentation paradigm: moving beyond rows and columns, Clinical Neurophysiology, 121, 1109-1120.

Schalk G, McFarland DJ, Hinterberger T, Birbaumer N, Wolpaw JR. (2004). BCI2000: a general-purpose brain-computer interface (BCI) system. IEEE Trans Biomed Eng. 51(6), pp.1034-43.

Schalk G. (2008). Brain-computer symbiosis. J Neural Eng. 5(1), Epub 2008 Jan 17. Review. 
Sellers, E.W., and Donchin, E. (2006). A P300-based brain-computer interface: initial tests by ALS patients. Clin. Neurophysiol., 117(3), pp.538-548.

Sellers, E. W., Vaughan, T. M. \& Wolpaw, J. R (submitted).A brain-computer interface for long-term independent home use. Amyotrophic Lateral Scleroses.

Taylor DM, Tillery SI, Schwartz AB. (2002). Direct cortical control of 3D neuroprosthetic devices. Science, 296(5574), pp.1829-32.

Townsend, G. T. et al.(submitted). A novel P300-based brain-computer interface stimulus presentation paradigm: moving beyond rows and columns. Clin Neurophysiol.

Velliste, M., Perel, S., Spalding, M.C., Whitford, A.S., Schwartz, A.B. (2008). Cortical control of a prosthetic arm for self-feeding. Nature 453(7198), pp. 1098-101.

Vidal, J.J. (1973). Toward direct brain-computer communication, Annu. Rev. Biophys. Bioeng., pp. 157-180.

Wessberg J, Stambaugh CR, Kralik JD, Beck PD, Laubach M, Chapin JK, Kim J, Biggs SJ, Srinivasan MA, Nicolelis MA. (2000). Real-time prediction of hand trajectory by ensembles of cortical neurons in primates. Nature, 408(6810), 361-5.

Wolpaw, J.R., Birbaumer, N., McFarland, D.J., Pfurtscheller, G., and Vaughan, T.M. (2002). Brain-computer interfaces for communication and control, Clin. Neurophysiol., 113, 6, pp. 767-791.

Wolpaw, J.R., McFarland, D.J., Vaughan, T.M., Schalk, G. (2003). The Wadsworth Center brain-computer interface (BCI) research and development program. IEEE Trans Neural Syst Rehabil Eng, 11(2), pp.204-7.

Zhang, H. (2009). A clinical study of motor imagery-based brain-computer interface for upper limb robotic rehabilitation, in Proc. EMBC, pp. 5981-5984. 


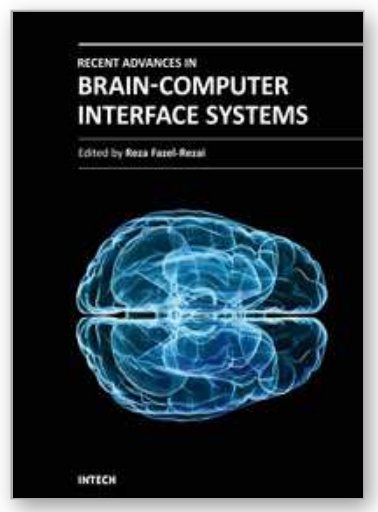

\author{
Recent Advances in Brain-Computer Interface Systems \\ Edited by Prof. Reza Fazel
}

ISBN 978-953-307-175-6

Hard cover, 222 pages

Publisher InTech

Published online 04, February, 2011

Published in print edition February, 2011

Brain Computer Interface $(\mathrm{BCl})$ technology provides a direct electronic interface and can convey messages and commands directly from the human brain to a computer. $\mathrm{BCl}$ technology involves monitoring conscious brain electrical activity via electroencephalogram (EEG) signals and detecting characteristics of EEG patterns via digital signal processing algorithms that the user generates to communicate. It has the potential to enable the physically disabled to perform many activities, thus improving their quality of life and productivity, allowing them more independence and reducing social costs. The challenge with $\mathrm{BCl}$, however, is to extract the relevant patterns from the EEG signals produced by the brain each second. Recently, there has been a great progress in the development of novel paradigms for EEG signal recording, advanced methods for processing them, new applications for $\mathrm{BCl}$ systems and complete software and hardware packages used for $\mathrm{BCl}$ applications. In this book a few recent advances in these areas are discussed.

\title{
How to reference
}

In order to correctly reference this scholarly work, feel free to copy and paste the following:

Christoph Guger et al. (2011). State-of-the-Art in BCI Research: BCl Award 2010, Recent Advances in BrainComputer Interface Systems, Prof. Reza Fazel (Ed.), ISBN: 978-953-307-175-6, InTech, Available from: $\mathrm{http}: / /$ www.intechopen.com/books/recent-advances-in-brain-computer-interface-systems/state-of-the-art-inbci-research-bci-award-2010

\section{INTECH}

open science | open minds

\section{InTech Europe}

University Campus STeP Ri

Slavka Krautzeka 83/A

51000 Rijeka, Croatia

Phone: +385 (51) 770447

Fax: +385 (51) 686166

www.intechopen.com

\section{InTech China}

Unit 405, Office Block, Hotel Equatorial Shanghai

No.65, Yan An Road (West), Shanghai, 200040, China

中国上海市延安西路65号上海国际贵都大饭店办公楼 405 单元

Phone: +86-21-62489820

Fax: $+86-21-62489821$ 
(C) 2011 The Author(s). Licensee IntechOpen. This chapter is distributed under the terms of the Creative Commons Attribution-NonCommercialShareAlike-3.0 License, which permits use, distribution and reproduction for non-commercial purposes, provided the original is properly cited and derivative works building on this content are distributed under the same license. 